\title{
Dynamic Factor Models with Infinite-Dimensional Factor Spaces: One-Sided Representations
}

\author{
Mario FORNI* \\ Università di Modena e Reggio Emilia, CEPR and RECent \\ Marc HALLin ${ }^{\dagger \ddagger}$ \\ ECARES, Université Libre de Bruxelles and ORFE, Princeton University \\ Marco LIPPI \\ Università di Roma La Sapienza and EIEF \\ Paolo ZAFFARONI ${ }^{\S}$ \\ Imperial College London and Università di Roma La Sapienza
}

November 25, 2014

\begin{abstract}
Factor model methods recently have become extremely popular in the theory and practice of large panels of time series data. Those methods rely on various factor models which all are particular cases of the Generalized Dynamic Factor Model (GDFM) introduced in Forni, Hallin, Lippi and Reichlin (2000). That paper, however, rests on Brillinger's dynamic principal components. The corresponding estimators are two-sided filters whose performance at the end of the observation period or for forecasting purposes is rather poor. No such problem arises with estimators based on standard principal components, which have been

${ }^{*}$ Research supported by the PRIN-MIUR Grant 2010J3LZEN-003.

${ }^{\dagger}$ Research supported by the Sonderforschungsbereich "Statistical modelling of nonlinear dynamic processes" (SFB 823) of the Deutsche Forschungsgemeinschaft, a Belgian Science Policy Office (20122017) Interuniversity Attraction Pole, and a Discovery Grant of the Australian Research Council.

${ }^{\ddagger}$ Académie Royale de Belgique and CentER, Tilburg University.

$\S$ Research supported by the ESRC Grant RES-000-22-3219.

${ }^{\top}$ Corresponding author, p.zaffaroni imperial.ac.uk
\end{abstract}


dominant in this literature. On the other hand, those estimators require the assumption that the space spanned by the factors has finite dimension. In the present paper, we argue that such an assumption is extremely restrictive and potentially quite harmful. Elaborating upon recent results by Anderson and Deistler (2008a, b) on singular stationary processes with rational spectrum, we obtain one-sided representations for the GDFM without assuming finite dimension of the factor space. Construction of the corresponding estimators is also briefly outlined. In a companion paper, we establish consistency and rates for such estimators, and provide Monte Carlo results further motivating our approach.

JEL subject classification : C0, C01, E0.

Key words and phrases : Generalized dynamic factor models. Vector processes with singular spectral density. One-sided representations for dynamic factor models.

\section{Introduction}

\subsection{Dynamic factor models}

High-dimensional factor model methods can be traced back to two seminal papers by Chamberlain (1983) and Chamberlain and Rothschild (1983). The recent and fastly growing literature on the subject, however, is starting with the contributions by Forni et al. (2000), Forni and Lippi (2001), Stock and Watson (2002a,b), Bai and Ng (2002) and Bai (2003). Fostered by their success in applications, factor model methods since then have attracted considerable attention. The recent literature in the area is so abundant that even a brief review is impossible here, and we restrict ourselves to a short and unavoidably somewhat subjective selection of "representative" references. Applications include (a) forecasting (Stock and Watson 2002a and b, Forni et al. 2005, Boivin and Ng 2006), (b) business cycle indicators and nowcasting (Cristadoro et al. 2005, Giannone et al. 2008, Altissimo et al. 2010), (c) structural macroeconomic analysis and monetary policy (Bernanke and Boivin 2003, Bernanke et al. 2005, Stock and Watson 2005, Giannone et al. 2005, Favero et al. 2005, Eickmeier 2007, Forni et al. 2009, Boivin et

al. 2009, Forni and Gambetti 2010b), (d) the analysis of financial markets (Corielli 
and Marcellino 2006, Ludvigson and Ng 2007 and 2009, Hallin et al. 2011), to quote only a few.

Apart for some minor features, most factor models considered in the literature are particular cases of the so-called Generalized Dynamic Factor Model (GDFM) introduced in Forni et al. (2000). Consider a countable set $\left\{x_{i t}\right\}, i \in \mathbb{N}$ of observable stationary stochastic processes. The GDFM relies on a decomposition of the form

$$
x_{i t}=\chi_{i t}+\xi_{i t}=b_{i 1}(L) u_{1 t}+b_{i 2}(L) u_{2 t}+\cdots+b_{i q}(L) u_{q t}+\xi_{i t}
$$

$i \in \mathbb{N}, t \in \mathbb{Z}$, where $\mathbf{u}_{t}=\left(u_{1 t} u_{2 t} \cdots u_{q t}\right)^{\prime}$ is a $q$-dimensional orthonormal unobservable white noise vector and $b_{i f}(L), i \in \mathbb{N}, f=1, \ldots, q$ are square-summable filters $(L$, as usual, stands for the lag operator). Moreover:

(I) $\mathbf{u}_{t}$ is orthogonal to $\xi_{i, t-k}$ for all $i \in \mathbb{N}, t \in \mathbb{Z}$ and $k \in \mathbb{Z}$;

(II) cross-covariances among the $\xi_{i t}$ 's are "weak".

By "weak", we mean that, while some cross-covariance among the $\xi$ 's is allowed, all sequences of weighted cross-sectional averages of the form $\sum_{i=1}^{n} w_{n i} \xi_{i t}$ such that $\lim _{n \rightarrow \infty} \sum_{i=1}^{n} w_{n i}^{2}=0$ tend to zero in mean square as $n \rightarrow \infty$ (the sequence of arithmetic averages $n^{-1} \sum_{i=1}^{n} \xi_{i t}$ being a particular case). ${ }^{1}$ Note that $\mathrm{E}\left(\xi_{i t}^{2}\right) \leq M$ for all $i$ and $\mathrm{E}\left(\xi_{i t} \xi_{j t}\right)=0$ for all $i \neq j$, is sufficient, but not necessary for (II) to hold (we refer to Section 2 for a detailed presentation and discussion).

Weak covariance of the $\xi_{i t}$ 's motivates calling them idiosyncratic, while the $\chi_{i t}$ 's, being driven by the low-dimensional vector of common shocks $u_{f t}, f=1,2 \ldots, q$, are called common components. The model implies that cross-covariances among the observable variables $x_{i t}$ are essentially accounted for by the common components $\chi_{i t}$.

The problem consists in recovering the unobserved common and idiosyncratic components $\chi_{i t}$ and $\xi_{i t}$, the common shocks $\mathbf{u}_{t}$ and the filters $b_{i f}(L)$, from finite realizations

\footnotetext{
${ }^{1}$ Weak cross-covariance among the $\xi$ 's, as opposed to cross-sectional orthogonality (that is, the much stronger assumption of no cross-covariances at all), is the reason for using the term "generalized" in the denomination of the GDFM. It constitutes a major difference with respect to the dynamic factor models studied in Sargent and Sims (1977), Geweke (1977), Quah and Sargent (1993), which, being based on a finite number $n$ of equations of the form (1.1), require strict cross-sectional orthogonality.
} 
$(i=1, \ldots, n ; t=1, \ldots, T)$ of the process $\left\{x_{i t}\right\}$, as $n$ and $T$ both tend to infinity. The main tool so far has been a principal component analysis (PC) of the variables $x_{i t}$, either standard or in the frequency domain (Brillinger's concept of dynamic principal components), depending on the assumptions made. The results obtained can be summarized as follows.

(i) The finite-dimension assumption. Most authors assume that, denoting by $\overline{\operatorname{span}}(\ldots)$ the space generated by a collection of random variables, ${ }^{2} \operatorname{span}\left(\chi_{i t}, i \in \mathbb{N}\right)$, for given $t$, has finite dimension $r$, where $r \geq q$. Under that assumption, model (1.1) can be rewritten as

$$
\begin{aligned}
x_{i t} & =\lambda_{i 1} F_{1 t}+\lambda_{i 2} F_{2 t}+\cdots+\lambda_{i r} F_{r t}+\xi_{i t} \\
\mathbf{F}_{t} & =\left(F_{1 t} \ldots F_{r t}\right)^{\prime}=\mathbf{N}(L) \mathbf{u}_{t},
\end{aligned}
$$

$i \in \mathbb{N}, t \in \mathbb{Z}$. This is fairly easy to prove, see Forni et al. (2009), Remark R, Section 2. In this case, we say that (1.1) admits a static representation. If, in addition, $\mathbf{N}(L)=\mathbf{N}(0)$, so that $\mathbf{F}_{t}$ is a white noise vector, then (1.1) is a static factor model. Criteria to determine $r$ consistently are given in Bai and $\mathrm{Ng}$ (2002) (see also Alessi et al. 2010). The vectors $\mathbf{F}_{t}$ and the loadings $\lambda_{i j}$ can be estimated consistently using the first $r$ standard principal components, see Stock and Watson (2002a,b), Bai and Ng (2002). Moreover, the second equation in (1.2) is usually specified as a singular VAR, so that (1.2) becomes

$$
\begin{aligned}
& x_{i t}=\lambda_{i 1} F_{1 t}+\lambda_{i 2} F_{2 t}+\cdots+\lambda_{i r} F_{r t}+\xi_{i t} \\
& \left(I-\mathbf{D}_{1} L-\mathbf{D}_{2} L^{2}-\ldots-\mathbf{D}_{p} L^{p}\right) \mathbf{F}_{t}=\mathbf{R} \mathbf{u}_{t},
\end{aligned}
$$

where the matrices $\mathbf{D}_{j}$ are $r \times r$ while $\mathbf{R}$ is $r \times q$. Under (1.3), Bai and $\mathrm{Ng}$ (2007) and Amengual and Watson (2007) provide consistent criteria to determine $q$. VAR estimation, and therefore, up to multiplication by an orthogonal matrix, estimation of $\mathbf{u}_{t}$ in (1.3), is standard.

\footnotetext{
${ }^{2}$ More precisely, $\overline{\operatorname{span}}\left(\zeta_{i}, i \in \mathbb{N}\right)$, where $\zeta_{i}$ belongs to the Hilbert space of square-summable random variables defined over some probability space, equipped with the corresponding $L^{2}$ norm, is the closed Hilbert space of all mean-square convergent linear combinations of the $\zeta_{i}$ 's and limits of convergent sequences thereof.
} 
(ii) Obtaining the static representation. Let us point out that (1.2) or (1.3) are convenient "reduced forms" of other, more explicitly dynamic, representations. For example, an interesting dynamic factor model is

$$
x_{i t}=\boldsymbol{\mu}_{i 0} \mathbf{f}_{t}+\boldsymbol{\mu}_{i 1} \mathbf{f}_{t-1}+\cdots+\boldsymbol{\mu}_{i p} \mathbf{f}_{t-p}+\xi_{i t},
$$

where $\mathbf{f}_{t}$ is a $q$-dimensional stationary vector, $\boldsymbol{\mu}_{i j}$ is $1 \times q$ and $\mathbf{D}(L) \mathbf{f}_{t}=\mathbf{u}_{t}$. Bai and Ng (2007) and Forni et al. (2009) show how (1.4) can be put in the form (1.2), or (1.3), and obtain the coefficients of (1.2), or (1.3), as functions of the coefficients of (1.4).

(iii) The dynamically unrestricted model. Using the frequency-domain principal components (Brillinger 1981), and without any finite-dimensional assumption of the form (1.2), Forni et al. (2000) obtain an estimator of the spectral density of the common components $\chi_{i t}$ and show how to consistently recover the common components themselves. Criteria to determine $q$ without assuming (1.2) or (1.3) are obtained in Hallin and Liška (2007) and Onatski (2009). Unfortunately, frequency-domain principal components produce estimators of the $\chi_{i t}$ 's that are based on two-sided filters, which hence cannot be used at the end of the sample or for prediction.

Due to that two-sidedness feature, the GDFM is seldom considered in practice, and finite-dimensional structure assumptions like (1.2) or (1.3) are made with almost no exception. Even the paper by Forni et al. (2005), which is based on the same frequency-domain approach as Forni et al. (2000), adopts a finite-dimension assumption for $\overline{\operatorname{span}}\left(\chi_{i t}, i \in \mathbb{N}\right)$ to obtain one-sided estimators. ${ }^{3}$

The moot point is that such assumptions are far from being innocuous. For instance, (1.2) is so restrictive that even the very elementary model

$$
x_{i t}=\frac{a_{i}}{1-\alpha_{i} L} u_{t}+\xi_{i t}
$$

\footnotetext{
${ }^{3}$ See also Altissimo et al. (2010), where the spectral-density principal-component approach is used in combination with the finite-dimensional assumption.
} 
where $q=1, u_{t}$ is scalar white noise, and the coefficients $\alpha_{i}$ are drawn from a uniform distribution over $(-1,1)$, is ruled out. Indeed, the space spanned, for a given $t$, by the common components $\chi_{i t}, i \in \mathbb{N}$, is easily seen to be infinite-dimensional. Infinitedimensional $\overline{\operatorname{span}}\left(\chi_{i t}, i \in \mathbb{N}\right)$ 's a fortiori occur if the AR common component in (1.5) is replaced by more general ARMA ones.

But even when the dimension of $\overline{\operatorname{span}}\left(\chi_{i t}, i \in \mathbb{N}\right)$ is finite there are interesting cases for which the dynamically unrestricted model and related methods provide an advantage over the static approach. Consider the model

$$
x_{i t}= \begin{cases}u_{t}+a u_{t-1}+\xi_{i t} & \text { if } i=1 \\ u_{t}+\xi_{i t} & \text { if } i>1,\end{cases}
$$

where $u_{t}$ is a scalar white noise, and suppose that we are interested in the first variable $x_{1 t}$. Of course this model, unlike (1.5), can be written in the static form (1.2), with $F_{1 t}=u_{t}$ and $F_{2 t}=u_{t-1}$. However, it does not fulfill a basic assumption of the static two-factor model, since $u_{t-1}$ is "non-pervasive" (see Assumption B.2, Section 2). As a consequence, the impulse response function of $x_{1 t}$, i.e. $1-a L$, cannot be obtained with the standard principal component method. By contrast, as shown in Section 2, model (1.6) can be easily accommodated within the dynamic approach proposed here. ${ }^{4}$

Such examples provide a strong theoretical motivation for solving the one-sidedness problem in model (1.1) without turning to the finite-dimension restriction and the related assumptions and methods. This is done in the present paper under assumptions that include rational spectral density for the common components $\chi_{i t}{ }^{5}$

On the other hand, we must also point out that, even when the finite-dimension assumption does not hold, model (1.2), or (1.3), can provide a good approximation to model (1.1), or, in empirical situations, with $n$ and $T$ given, a good fit or a good performance in forecasting. These problems are not studied in the present paper, in which we only deal with representation issues and make use of population covariances

\footnotetext{
${ }^{4}$ Model (1.6) is just a stylized example of a situation in which some of the lags of the common shocks are non-pervasive.

${ }^{5}$ Some of the results presented her have been outlined, without proofs, in a very preliminary version in Forni and Lippi (2011).
} 
and spectral densities. The companion paper, Forni, Hallin, Lippi and Zaffaroni (2014), gives a detailed definition of the estimators corresponding to the construction of the present paper, studies their consistency rates, and compares, by means of Monte Carlo experiments, the performance of the static and the dynamic approach. A brief outline of these results is given in Section 4.5.

\subsection{Outline of the paper}

Instead of finite-dimensional assumptions of the form (1.2) or (1.3), we impose the much milder condition that the common components have a rational spectral density, that is, each filter $b_{i f}(L)$ in $(1.1)$ is a ratio of polynomials in $L$. More precisely, we assume the following representation for the common components:

$$
\chi_{i t}=\frac{c_{i 1}(L)}{d_{i 1}(L)} u_{1 t}+\frac{c_{i 2}(L)}{d_{i 2}(L)} u_{2 t}+\cdots+\frac{c_{i q}(L)}{d_{i q}(L)} u_{q t},
$$

where

$$
c_{i f}(L)=c_{i f, 0}+c_{i f, 1} L+\ldots+c_{i f, s_{1}} L^{s_{1}} \quad \text { and } \quad d_{i f}(L)=d_{i f, 0}+d_{i f, 1} L+\ldots+d_{i f, s_{2}} L^{s_{2}},
$$

$f=1,2, \ldots, q$. The assumption that $s_{1}$ and $s_{2}$, the degrees of $c_{i f}(L)$ and $d_{i f}(L)$ respectively, are assumed to be independent of $i$ is very convenient, though not necessary. As for the idiosyncratic components we do not make any parametric assumptions, nor restrict their cross-covariance structure - except of course for the "weak cross-correlation assumption" that characterizes idiosyncrasy, as described above. Our model, in that sense, is a semiparametric one, with a huge nuisance; in particular, the autocorrelation structures of idiosyncratic components remain completely unspecified.

We show that, for generic values of the parameters $c_{i f, k}$ and $d_{i f, k}$ (i.e. apart from a subset that is negligible, in a sense to be specified in Section 2), the infinite-dimensional common-component vector $\chi_{t}=\left(\begin{array}{llllll}\chi_{1 t} & \chi_{2 t} & \cdots & \chi_{n t} & \cdots\end{array}\right)^{\prime}$ admits a block-structure au- 
toregressive representation

$$
\left(\begin{array}{ccccc}
\mathbf{A}^{1}(L) & 0 & \cdots & 0 & \cdots \\
0 & \mathbf{A}^{2}(L) & \cdots & 0 & \\
& & \ddots & & \\
0 & 0 & \cdots & \mathbf{A}^{k}(L) & \\
\vdots & & & & \ddots
\end{array}\right) \chi_{t}=\left(\begin{array}{c}
\mathbf{R}^{1} \\
\mathbf{R}^{2} \\
\vdots \\
\mathbf{R}^{k} \\
\vdots
\end{array}\right) \mathbf{u}_{t}
$$

where each $\mathbf{A}^{k}(L)$ is a $(q+1) \times(q+1)$ polynomial matrix with finite degree and $\mathbf{R}^{k}$ is $(q+1) \times q$. Denoting by $\underline{\mathbf{A}}(L)$ and $\underline{\mathbf{R}}$ the (infinite) matrices on the left- and right-hand sides of (1.8), respectively, and defining $\mathbf{x}_{t}$ and $\boldsymbol{\xi}_{t}$ in analogy with $\chi_{t}$, we obtain

$$
\mathbf{Z}_{t}=\underline{\mathbf{R}} \mathbf{u}_{t}+\underline{\mathbf{A}}(L) \boldsymbol{\xi}_{t}
$$

where $\mathbf{Z}_{t}=\underline{\mathbf{A}}(L) \mathbf{x}_{t}$, and, lastly,

$$
\mathbf{z}_{t}=\underline{\mathbf{r}} \mathbf{u}_{t}+\phi_{t}
$$

which results from (1.9) by normalization (both sides of the $i$-th equation are divided by the standard deviation of $Z_{i t}$ ). This is a factor model with a representation of the form (1.2) and $\mathbf{F}_{t}=\mathbf{u}_{t}$ - thus, according to the definition given in Section 1.1, a static factor model.

Some comments on (1.8)-(1.10) are in order.

(i) We can rewrite (1.8) as $\mathbf{A}^{k}(L) \boldsymbol{\chi}_{t}^{k}=\mathbf{R}^{k} \mathbf{u}_{t}, k \in \mathbb{N}$, where the vectors $\chi_{t}^{k}$ are the $(q+1)$-dimensional subvectors

$$
\left(\begin{array}{llll}
\chi_{1 t} & \chi_{2 t} & \cdots & \chi_{q+1, t}
\end{array}\right),\left(\chi_{q+2, t} \chi_{q+3, t} \cdots \chi_{2(q+1), t}\right), \cdots
$$

Thus (1.8) is made up of (a) obtaining an autoregressive representation for each of the vectors $\chi_{t}^{k}$, and then (b) knitting together such autoregressive representations.

(ii) As regards (a), each of the subvectors has dimension $(q+1)$ and rank $q$ (i.e. its spectral density has $\operatorname{rank} q$ for all $\theta \in[-\pi \pi]$ ), and is therefore singular (i.e. its dimension is greater than its rank). For singular (or reduced-rank) vectors, 
with rational spectral density, existence of a finite-degree autoregressive representation, for generic values of the parameters, has been proved in Anderson and Deistler (2008a, b). We contribute to this literature by showing that, when the dimension is equal to $q+1$, the minimum-lag autoregressive representation is generically unique. As regards (b), obtaining the same $\mathbf{u}_{t}$ for all the vectors $\chi_{t}^{k}$ requires the additional assumption that, for each $k, \overline{\operatorname{span}}\left(\chi_{t-h}^{k}, h \geq 0\right)=$ $\overline{\operatorname{span}}\left(\chi_{t-h}, h \geq 0\right)$. We will motivate this restriction by a genericity argument.

(iii) The matrices $\mathbf{A}^{k}(L)$ and $\mathbf{R}^{k}$ can be obtained starting with the spectral density matrix of the observable variables $x_{i t}$. The vector $\mathbf{z}_{t}$ results from the application of one-sided filters to the variables $x_{i t}$, see (1.10). Lastly, $\mathbf{u}_{t}$ can be obtained using the first $q$ principal components of the variables $z_{i t}$, i.e. only current values of the variables $z_{i t}$. Our procedure thus solves the one-sidedness problem.

(iv) Moreover, the matrices $\mathbf{A}^{k}(L)$ and $\mathbf{R}^{k}$, which are $(q+1) \times(q+1)$ and $(q+1) \times q$ respectively, result from separate low-dimensional calculations. Thus we do not run into "curse of dimensionality" problems.

In Section 2, we state the main assumptions underlying the GDFM and review some basic results from previous literature. In Section 3, we prove some general results on stochastic vectors that are infinite-dimensional with finite rank, like $\boldsymbol{\chi}_{t}$, under the assumption of rational spectral density. Rational spectral density is assumed for $\chi_{t}$ throughout the paper. In Section 4, we present results on autoregressive representations of singular stochastic vectors. Such results are then used to construct the blockwise autoregressive representation (1.8) for $\chi_{t}$ and to transform the original variables $x_{i t}$ into another set of variables for which a static factor model holds. Lastly, we briefly outline the correspondence between our representation result here and the estimation procedure that we study in the companion paper Forni et al. (2014). Section 5 concludes. 


\section{Main assumptions and background results}

\section{$2.1 \quad$ Notation}

The GDFM (1.1) can be thought of as (i) a double-indexed stochastic process $\left\{x_{i t}, i \in\right.$ $\mathbb{N}, t \in \mathbb{Z}\}$, (ii) a family of stationary processes $\left\{x_{i t}, t \in \mathbb{Z}\right\}$ indexed by $i \in \mathbb{N}$, or (iii) a stationary family of cross-sections $\left\{x_{i t}, i \in \mathbb{N}\right\}$ indexed by $t \in \mathbb{Z}$, i.e. a stationary infinite-dimensional stochastic process ${ }^{6}$. We find the third option convenient, and accordingly write $\mathbf{x}_{t}$ for $\left(x_{1 t} x_{2 t} \cdots x_{n t} \cdots\right)^{\prime}$. The notation $\chi_{t}, \boldsymbol{\xi}_{t}$ and $\mathbf{x}_{t}=\chi_{t}+\boldsymbol{\xi}_{t}$ is used in a similar way, with obvious componentwise counterparts. Associated with this infinite-dimensional vector notation, we also consider infinite-dimensional matrices, such as $\underline{\mathbf{A}}(L)$ or $\underline{\mathbf{R}}$ (see (1.10)), which are $\infty \times \infty$ and $\infty \times q$, respectively. Also, defining $\underline{\mathbf{b}}(L)$ as the $\infty \times q$ matrix with $(i, f)$-entry $b_{i f}(L),(1.1)$ is rewritten as $\mathbf{x}_{t}=\underline{\mathbf{b}}(L) \mathbf{u}_{t}+\boldsymbol{\xi}_{t}$. The reader will easily check that we never produce infinite sums of products, so that our infinite-dimensional matrices are no more than a notational convenience. All infinite-dimensional matrices are underlined, while their finite-dimensional submatrices are not. In particular, $\mathbf{A}_{s}(L)$ denotes the $s \times s$ upper left submatrix of $\underline{\mathbf{A}}(L), \mathbf{b}_{s}(L)$ and $\mathbf{R}_{s}$ the $s \times q$ upper submatrices of $\underline{\mathbf{b}}(L)$ and $\underline{\mathbf{R}}$, respectively.

Given the infinite-dimensional process $\mathbf{y}_{t}=\left(\begin{array}{llll}y_{1 t} & y_{2 t} & \cdots & y_{n t} \\ \cdots\end{array}\right)^{\prime}$, we use the following notation:

(i) $\mathbf{y}_{s t}$ is the $s$-dimensional process $\left(y_{1 t} y_{2 t} \cdots y_{s t}\right)^{\prime}$;

(ii) $\mathcal{H}^{y}=\overline{\operatorname{span}}\left(y_{i t}, i \in \mathbb{N}, t \in \mathbb{Z}\right), \quad \mathcal{H}^{y_{s}}=\overline{\operatorname{span}}\left(y_{i t}, 1 \leq i \leq s, t \in \mathbb{Z}\right)$;

(iii) $\mathcal{H}_{t}^{y}=\overline{\operatorname{span}}\left(y_{i \tau}, i \in \mathbb{N}, \tau \leq t\right), \quad \mathcal{H}_{t}^{y_{s}}=\overline{\operatorname{span}}\left(y_{i \tau}, 1 \leq i \leq s, \tau \leq t\right)$.

If $\mathbf{y}_{t}$ is $s$-dimensional we use the notation $\mathcal{H}^{y}=\overline{\operatorname{span}}\left(y_{i t}, i \leq s, t \in \mathbb{Z}\right), \mathcal{H}_{t}^{y}=$ $\overline{\operatorname{span}}\left(y_{i \tau}, i \leq s, \tau \leq t\right)$ (we never need sub-vectors of finite-dimensional vectors).

\footnotetext{
${ }^{6}$ For an introduction to infinite-dimensional stationary stochastic processes, their spectral representation and prediction theory, see Salehi (1981). Some results on infinite-dimensional processes are proved in the present paper. However, as we assume rational spectral density and finite rank, see Section 3 , our proofs only need straightforward generalizations of results holding in the finite-dimensional case.
} 
It is convenient, though not necessary, to assume throughout the paper that all white-noise vectors are orthonormal.

\subsection{Basic assumptions}

All the stochastic variables $x_{i t}, \chi_{i t}$ and $\xi_{i t}$ below have mean zero and finite variance.

Assumption A.1 For all $n \in \mathbb{N}$, the vector $\mathbf{x}_{n t}$ is weakly stationary (stationary henceforth), and has a spectral density (an absolutely continuous spectral measure).

Denote by $\boldsymbol{\Sigma}_{n}^{x}(\theta)$, with entries $\sigma_{i j}^{x}(\theta), i, j \in \mathbb{N}, \theta \in[-\pi \pi]$, the nested spectral density matrices of the vectors $\mathbf{x}_{n t}=\left(\begin{array}{llll}x_{1 t} & x_{2 t} & \cdots & x_{n t}\end{array}\right)^{\prime}$. The matrix $\Sigma_{n}^{x}(\theta)$ is Hermitian, non-negative definite and has therefore non-negative real eigenvalues for all $\theta \in[-\pi \pi]$. Denote by $\lambda_{n j}^{x}(\theta)$ the $j$-th eigenvalue, in decreasing order, of $\Sigma_{n}^{x}(\theta)$, and let $\bar{\lambda}_{f}^{x}(\theta)=\sup _{n \in \mathbb{N}} \lambda_{n f}^{x}(\theta)$. The notation $\boldsymbol{\Sigma}_{n}^{\chi}(\theta), \sigma_{i j}^{\chi}(\theta), \lambda_{n j}^{\chi}(\theta), \bar{\lambda}_{f}^{\chi}(\theta), \boldsymbol{\Sigma}_{n}^{\xi}(\theta), \sigma_{i j}^{\xi}(\theta)$, $\lambda_{n j}^{\xi}(\theta)$, and $\bar{\lambda}_{f}^{\xi}(\theta)$ is used in a similar way. Our second assumption is

Assumption A.2 There exists a positive integer $q$ such that $(i) \bar{\lambda}_{q}^{x}(\theta)=\infty$ for almost all $\theta$ in $[-\pi \pi]$, and (ii) $\bar{\lambda}_{q+1}^{x}(\theta)$ is essentially bounded, i.e. there exists a real $B^{x}$ such that $\bar{\lambda}_{q+1}^{x}(\theta) \leq B^{x}$ almost everywhere in $[-\pi \pi]$.

Forni and Lippi (2001) prove that

Theorem A Assumptions A.1 and A.2 imply that $\mathbf{x}_{t}$ can be represented as in (1.1), i.e.

$$
\mathbf{x}_{t}=\chi_{t}+\xi_{t}=\underline{\mathbf{b}}(L) \mathbf{u}_{t}+\xi_{t}
$$

where $\underline{\mathbf{b}}(L)$ is an $\infty \times q$ matrix of square-summable filters, $\mathbf{u}_{t}$ is a $q$-dimensional orthonormal white noise. Moreover,

(i) $\boldsymbol{\xi}_{n t}$ satisfies Assumption A.1, and $\bar{\lambda}_{1}^{\xi}(\theta)$ is essentially bounded, i.e. there exists a real $B^{\xi}$ such that $\bar{\lambda}_{1}^{\xi}(\theta) \leq B^{\xi}$ almost everywhere in $[-\pi \pi]$;

(ii) $\chi_{t}$ satisfies $A .1$ and $\bar{\lambda}_{q}^{\chi}(\theta)=\infty$ almost everywhere in $\theta$ in $[-\pi \pi]$ (note that $\bar{\lambda}_{q+s}^{\chi}(\theta)=0$ a.e. in $[-\pi \pi]$ for all $\left.s>0\right)$;

(iii) $\boldsymbol{\xi}_{t}$ and $\mathbf{u}_{t-k}$ are uncorrelated for all $t \in \mathbb{Z}$ and $k \in \mathbb{Z}$; 
(iv) the components $\chi_{i t}$ and $\xi_{i t}$ are unique.

Conversely, if $\mathbf{x}_{t}$ can be represented as in (2.1) with $\boldsymbol{\chi}_{t}$ and $\boldsymbol{\xi}_{t}$ fulfilling (i), (ii) and (iii), then $\mathbf{x}_{t}$ satisfies Assumptions A.1 and A.2.

An infinite-dimensional vector satisfying (i) is called an idiosyncratic vector.

Under the restriction that the dimension of $\overline{\operatorname{span}}\left(\chi_{i t}, i \in \mathbb{N}\right)$ is finite, so that the model has representation (1.2), or (1.3), the basic assumptions are:

Assumption B.1 Same as A.1.

Assumption B.2 Let $\Gamma_{n}^{x}$ be the variance-covariance matrix of $\mathbf{x}_{n t}$, $\mu_{n j}^{x}$ its $j$-th eigenvalue and $\bar{\mu}_{j}^{x}=\sup _{n \in \mathbb{N}} \mu_{n j}^{x}$. There exists a positive $r$ such that $(i) \bar{\mu}_{r}^{x}=\infty$, and (ii) $\bar{\mu}_{r+1}^{x}<\infty$.

Theorem B (Chamberlain and Rothschild, 1983) Assumptions B.1 and B.2 imply that $\mathbf{x}_{t}$ can be represented as

$$
x_{i t}=\chi_{i t}+\xi_{i t}=\lambda_{i 1} F_{1 t}+\lambda_{i 2} F_{2 t}+\cdots+\lambda_{i r} F_{r t}+\xi_{i t}
$$

where $\mathbf{F}_{t}$ is a weakly stationary $r$-dimensional vector. Moreover,

(i) $\boldsymbol{\xi}_{t}$ satisfies Assumption B.1 and $\bar{\mu}_{1}^{\xi}<\infty$;

(ii) $\chi_{t}$ satisfies Assumption B.1 and $\bar{\mu}_{r}^{\chi}=\infty$ (note that $\bar{\mu}_{r+s}^{\chi}=0$ for all $\left.s>0\right)$;

(iii) $\boldsymbol{\xi}_{t}$ and $\mathbf{F}_{t}$ are uncorrelated for all $t \in \mathbb{Z}$;

(iv) the integer $r$ and the components $\chi_{i t}$ and $\xi_{i t}$ are unique.

Conversely, if $\mathbf{x}_{t}$ can be represented as in (2.2) with $\boldsymbol{\chi}_{t}$ and $\boldsymbol{\xi}_{t}$ fulfilling (i), (ii) and (iii), then $\mathbf{x}_{t}$ satisfies Assumptions B.1 and B.2.

Under Assumptions A1 and A2, plus some technical assumptions, model (1.1) can be estimated using the (estimated) spectral density of the $x$ 's, see Forni et al. 2000. As mentioned in the Introduction, Hallin and Liška (2007) and Onatski (2009) provide criteria to determine $q$, while Forni et al. (2000) construct a two-sided estimator for $\chi_{t}$. All these papers use spectral techniques. A combination of spectral and time-domain 
techniques is used in the present paper to obtain a one-sided representation. For the corresponding one-sided estimator, see Forni et al. (2014).

Under the finite-dimension restriction and Assumptions B.1 and B.2, plus some technical assumptions, model (1.2), or (1.3), can be estimated using the variancecovariance matrix of the $x$ 's: seminal papers are Bai and $\mathrm{Ng}$ (2002), providing criteria to determine $r$, and Stock and Watson (2002a, b), constructing an estimator for $\mathbf{F}_{t}$. Bai and $\mathrm{Ng}$ (2007) develop tests for the number of dynamic factors $q$ in model (1.3) without resorting to spectral techniques.

Example (1.6) in the Introduction, i.e. $x_{1 t}=u_{t}+a u_{t-1}+\xi_{1 t}, x_{i t}=u_{t}+\xi_{i t}$ for $i>1$, nicely highlights a noticeable difference between Assumptions A.2 and B.2, corresponding to a basic difference between the dynamic and the static approaches. Using the dynamic approach, we see that the first eigenvalue of the spectral density matrix diverges and Asssumption A.2 is fulfilled with $q=1$. Hence the common component of the first variable is $u_{t}+a u_{t-1}$ and its idiosyncratic component is $\xi_{1 t}$. Using the techniques of the present paper, the (bivariate) VAR corresponding to the first block in equation (1.8) is

$$
\left(\begin{array}{cc}
1 & -a L \\
0 & 1
\end{array}\right)\left(\begin{array}{l}
\chi_{1 t} \\
\chi_{2 t}
\end{array}\right)=\left(\begin{array}{l}
1 \\
1
\end{array}\right) u_{t},
$$

while all other bivariate blocks $\left(x_{2 j+1, t}, x_{2(j+1), t}\right)(j=1,2, \ldots)$ have $\mathbf{A}^{k}(L)=\mathbf{I}_{2}$ and $\mathbf{R}^{k}=\left(\begin{array}{ll}1 & 1\end{array}\right)^{\prime}$, so that we obtain the correct representation $(1+a L) u_{t}$ for $\chi_{1 t}$, that is, the correct response of $x_{1 t}$ to the common shock $u_{t}$.

On the other hand, using the static approach, we find that only the first eigenvalue of the variance-covariance matrix diverges. Assumption B.2 is fulfilled with $r=1$, namely, by Theorem B, the model has a static factor representation with just one factor, i.e. $u_{t}$, whereas $u_{t-1}$, being non-pervasive, is not a common factor. The common component of the first variable is $u_{t}$ and the term $a u_{t-1}$ is absorbed by the idiosyncratic component, so that the model fails to correctly represent the reaction of $x_{1 t}$ to the shock $u_{t}{ }^{7}$

\footnotetext{
${ }^{7}$ The resulting lagged covariance between the common and the idiosyncratic component of $x_{1 t}$ is ignored within the static approach.
} 


\section{Infinite-dimensional processes with finite rank}

Of course, uniqueness of $\boldsymbol{\chi}_{t}$ and $\boldsymbol{\xi}_{t}$ in (2.1) does not imply that $\mathbf{u}_{t}$ or $\underline{\mathbf{b}}(L)$ are unique. Alternative representations are $\chi_{t}=[\underline{\mathbf{b}}(L) \mathbf{Q}]\left[\mathbf{Q}^{\prime} \mathbf{u}_{t}\right]=\underline{\mathbf{c}}(L) \mathbf{v}_{t}$, where $\mathbf{Q}$ is an arbitrary $q \times q$ orthogonal matrix, or, more generally, $\chi_{t}=[\underline{\mathbf{b}}(L) \mathbf{Q}(L)]\left[\left(\mathbf{Q}^{\prime}(F) \mathbf{u}_{t}\right]=\underline{\mathbf{d}}(L) \mathbf{w}_{t}\right.$, where $F=L^{-1}$ and $\mathbf{Q}\left(e^{-i \theta}\right) \mathbf{Q}^{\prime}\left(e^{i \theta}\right)=\mathbf{I}_{q}$ for almost all $\theta$ in $[-\pi \pi]$.

More importantly, Theorem A does not ensure that $\chi_{t}$ admits a one-sided movingaverage representation, i.e., a representation of the form $\chi_{t}=\underline{\mathbf{e}}(L) \mathbf{w}_{t}$, where $\mathbf{w}_{t}$ is $q$-dimensional orthonormal white noise and $\underline{\mathbf{e}}(L)=\underline{\mathbf{e}}_{0}+\underline{\mathbf{e}}_{1} L+\cdots$. For example, if

$$
\chi_{i t}=u_{t+i-1}
$$

where $u_{t}$ is one-dimensional white noise $(q=1)$, then statement (ii) of Theorem A holds true, so that $\chi_{t}$ is the common component of some process $\mathbf{x}_{t}$ satisfying A.1 and A.2, but $\chi_{t}$ has no one-sided representations (this is quite obvious from Lemma 1 below). ${ }^{8}$

The existence of one-sided moving average representations of infinite-dimensional stochastic vectors is analyzed in Lemmas 1 and 2 under the assumptions of rational spectral density and finite rank. A precise statement of those lemmas requires giving some further definitions and recalling a few results on rational-spectrum finitedimensional stochastic vectors.

Definition 1 Consider the infinite-dimensional process $\mathbf{y}_{t}=\left(\begin{array}{llllll}y_{1 t} & y_{2 t} & \cdots & y_{n t} & \cdots\end{array}\right)^{\prime}$. Assume that $\mathbf{y}_{t}$ fulfills Assumption A.1. We say that $\mathbf{y}_{t}$ has rank $q$ if there exists a positive integer s such that $\operatorname{rank}\left(\Sigma_{n}^{y}(\theta)\right)=q$, for $n \geq s$ and almost all $\theta$ in $[-\pi \pi]$.

Definition 2 Let $\mathbf{y}_{t}$ denote an infinite-dimensional stationary stochastic vector with a moving average representation

$$
\mathbf{y}_{t}=\underline{\mathbf{b}}(L) \mathbf{v}_{t},
$$

\footnotetext{
${ }^{8}$ The possibility that $\boldsymbol{\chi}_{t}$ has no one-sided representations arises here from infinite dimension. This bears no relationship with the possible non-existence of one-sided representations for finite-dimensional processes. For example, a scalar process whose spectral density vanishes in $\left[\begin{array}{ll}-1 & 1\end{array}\right]$ and is positive elsewhere has no one-sided representations, see e.g. Pourahmadi (2001), Theorem 10.5, p. 361.
} 
where $\mathbf{v}_{t}$ is q-dimensional orthonormal white noise and $\underline{\mathbf{b}}(L)$ is an $\infty \times q$ squaresummable filter. We say that (3.2) is a fundamental representation if (1) $\underline{\mathbf{b}}(L)$ is one-sided, and (2) $\mathbf{v}_{t}$ belongs to $\mathcal{H}_{t}^{y}$. In that case, we also say that the white noise $\mathbf{v}_{t}$ is fundamental for $\mathbf{y}_{t}$. Note that if $\mathbf{v}_{t}$ is fundamental for $\mathbf{y}_{t}$, then $\mathcal{H}_{t}^{v}=\mathcal{H}_{t}^{y}$.

Now suppose that $\mathbf{y}_{t}$ is $n$-dimensional with representation

$$
\mathbf{y}_{t}=\mathbf{b}(L) \mathbf{v}_{t}
$$

where $\mathbf{v}_{t}$ is $q$-dimensional orthonormal white noise and $\mathbf{b}(L)$ is an $n \times q$ squaresummable filter. Fundamentalness of (3.3) and $\mathbf{v}_{t}$ are defined as in Definition 2. Moreover,

(I) if (3.3) is fundamental, then $n \geq q$. Moreover, if $\mathbf{y}_{t}=\mathbf{c}(L) \mathbf{w}_{t}$, where $\mathbf{w}_{t}$ is orthonormal, is another fundamental representation, then $\mathbf{w}_{t}$ has dimension $q$, $\mathbf{c}(L)=\mathbf{b}(L) \mathbf{Q}$ and $\mathbf{w}_{t}=\mathbf{Q}^{\prime} \mathbf{v}_{t}$, where $\mathbf{Q}$ is a $q \times q$ orthogonal matrix (Rozanov 1967, pp. 56-57);

(II) if (3.3) is fundamental, then $\operatorname{rank}(\mathbf{b}(z))=q$ for all complex $z$ such that $|z|<1$ (Rozanov 1967, p. 63, Remark 3). In particular, $\operatorname{rank}\left(b_{0}\right)=\operatorname{rank}(\mathbf{b}(0))=q$.

A finite-dimensional stationary process with a spectral density does not necessarily possess a fundamental representation (see footnote 8). However,

(III) if $\mathbf{y}_{t}$ has rational spectral density, then it has fundamental representations. If $\mathbf{y}_{t}=\mathbf{b}(L) \mathbf{v}_{t}$ is one of them, $\mathbf{v}_{t}$ being $q$-dimensional orthonormal white noise, then the entries of $\mathbf{b}(L)$ are rational functions of $L$ (Rozanov 1967, Chapter I, Section 10; Hannan 1970, pp. 62-67);

$\left(\mathrm{II}^{\prime}\right)$ suppose that $\mathbf{y}_{t}$ has rational spectral density, that $\mathbf{y}_{t}=\mathbf{b}(L) \mathbf{v}_{t}$, where $\mathbf{b}(L)$ is $n \times q$, rational, square-summable and one-sided, $\mathbf{v}_{t}$ is $q$-dimensional orthonormal white noise, and that $\operatorname{rank}(\mathbf{b}(z))=q$ for all $z$ such that $|z|<1$ : then, $\mathbf{y}_{t}=\mathbf{b}(L) \mathbf{v}_{t}$ is fundamental (Hannan, 1970, pp. 62-67).

We say that the infinite-dimensional process $\mathbf{y}_{t}$ has rational spectral density if $\mathbf{y}_{n t}$ has rational spectral density for all $n$. 
Lemma 1 Suppose that the infinite-dimensional process $\mathbf{y}_{t}$ has rational spectral density and rank $q$. The following statements are equivalent:

(i) $\mathbf{y}_{t}$ has a one-sided rational moving average representation $\mathbf{y}_{t}=\underline{\mathbf{b}}(L) \mathbf{v}_{t}$ (the entries of $\underline{\mathbf{b}}(L)$ are rational functions of $L)$, where $\mathbf{v}_{t}$ is q-dimensional orthonormal white noise.

(ii) There exists a positive integer s such that $\mathcal{H}_{t}^{y_{s}}=\mathcal{H}_{t}^{y}$.

Proof. Assume (ii). By (III) there exists a one-sided rational fundamental representation for $y_{s t}$, denote it by $\mathbf{y}_{s t}=\mathbf{b}_{s}(L) \mathbf{v}_{t}$. We have $\mathcal{H}_{t}^{y_{s}}=\mathcal{H}_{t}^{v}$. By assumption, $y_{s+k, t} \in \mathcal{H}_{t}^{y_{s}}$ and, therefore, $y_{s+k, t} \in \mathcal{H}_{t}^{v}$, so that

$$
\mathbf{y}_{s t}=\mathbf{b}_{s}(L) \mathbf{v}_{t} \quad \text { and } \quad y_{s+k, t}=b_{s+k}(L) \mathbf{v}_{t} .
$$

The white noise $\mathbf{v}_{t}$ is fundamental for $\mathbf{y}_{s t}$, hence also for $\left(\mathbf{y}_{s t}^{\prime} y_{s+k, t}\right)^{\prime}$. Thus representation (3.4) is fundamental, so that, by (III), $b_{s+k}(L)$ must be rational. The conclusion follows. Assume now that (i) holds. We say that $\beta$ is a zero of $\underline{\mathbf{b}}(L)$ if the determinants of the $q \times q$ submatrices of $\underline{\mathbf{b}}(\beta)$ all vanish. Assume that $\alpha$ is a zero of $\underline{\mathbf{b}}(L)$ and that $|\alpha|<1$. There exists a unitary $q \times q$ matrix $\mathbf{B}_{\alpha}$ such that all the entries of the first column of $\underline{\mathbf{b}}(L) \mathbf{B}_{\alpha}$ vanish at $\alpha$. Defining $\gamma_{\alpha}(L)$ as the $q \times q$ diagonal matrix with diagonal entries $\left((1-\alpha L)(L-\alpha)^{-1} 1 \cdots 1\right)$, we have

$$
\mathbf{y}_{t}=\left[\underline{\mathbf{b}}(L) \mathbf{B}_{\alpha} \gamma_{\alpha}(L)\right]\left[\gamma_{\tilde{\alpha}}\left(L^{-1}\right) \tilde{\mathbf{B}}_{\alpha} \mathbf{v}_{t}\right]=\underline{\mathbf{c}}(L) \mathbf{w}_{t},
$$

where a tilde denotes transposition and conjugation. This is an alternative one-sided rational representation in which the multiplicity of $\alpha$ as a zero of the matrix polynomial has decreased by one unit. Because a zero of $\underline{\mathbf{b}}(L)$ is a zero of $\mathbf{b}_{q}(L)$, with a finite number of iterations we obtain a rational representation, $\mathbf{y}_{t}=\underline{\mathbf{d}}(L) \mathbf{z}_{t}$, say, such that $\underline{\mathbf{d}}(L)$ has no zeros of modulus less than one. For the same reason, there exists an integer $s$ such that $\mathbf{d}_{s}(L)$ has no zeros of modulus less than one. By $\left(\mathrm{II}^{\prime}\right), \mathbf{y}_{s t}=\mathbf{d}_{s}(L) \mathbf{z}_{t}$ is fundamental for $\mathbf{y}_{s t}$ and therefore for $\mathbf{y}_{t}$.

Q.E.D.

Lemma 2 Suppose that the infinite-dimensional process $\mathbf{y}_{t}$ has rational spectral density and rank $q$. Then, 
(i) if $\mathbf{y}_{t}$ has a one-sided rational representation $\mathbf{y}_{t}=\underline{\mathbf{b}}(L) \mathbf{v}_{t}$, then $\mathbf{y}_{t}$ has a fundamental (rational) representation;

(ii) if $\mathbf{y}_{t}=\underline{\mathbf{b}}(L) \mathbf{v}_{t}$ and $\mathbf{y}_{t}=\underline{\mathbf{c}}(L) \mathbf{w}_{t}$ are fundamental, with $\mathbf{v}_{t}$ and $\mathbf{w}_{t}$ q-dimensional and orthonormal, then $\underline{\mathbf{c}}(L)=\underline{\mathbf{b}}(L) \mathbf{Q}$ and $\mathbf{w}_{t}=\mathbf{Q}^{\prime} \mathbf{v}_{t}$, where $\mathbf{Q}$ is some $q \times q$ orthogonal matrix;

(iii) if $\mathbf{y}_{t}=\underline{\mathbf{b}}(L) \mathbf{v}_{t}=\underline{\mathbf{b}}_{0} \mathbf{v}_{t}+\underline{\mathbf{b}}_{1} \mathbf{v}_{t-1}+\cdots$ is fundamental, then $\underline{\mathbf{b}}_{0}$ has rank $q$.

Proof. Statement (i) is part of the proof of Lemma 1. As for (ii), suppose that $\mathbf{y}_{t}=\underline{\mathbf{b}}(L) \mathbf{v}_{t}$ and $\mathbf{y}_{t}=\underline{\mathbf{c}}(L) \mathbf{w}_{t}$ both are fundamental. By Lemma 1, there exists $s$ such that $\mathcal{H}_{t}^{y_{s}}=\mathcal{H}_{t}^{y}$. As a consequence, both $\mathbf{v}_{t}$ and $\mathbf{w}_{t}$ belong to $\mathcal{H}_{t}^{y_{s}}$, and therefore are fundamental for $\mathbf{y}_{s t}$. This implies that $\mathbf{w}_{t}=\mathbf{Q}^{\prime} \mathbf{v}_{t}$, where $\mathbf{Q}$ is orthogonal. Thus $\mathbf{y}_{t}=\underline{\mathbf{c}}(L) \mathbf{w}_{t}=\left[\underline{\mathbf{c}}(L) \mathbf{Q}^{\prime}\right] \mathbf{v}_{t}=\underline{\mathbf{b}}(L) \mathbf{v}_{t}$. As $\mathbf{v}_{t}$ is orthonormal white noise, we have $\underline{\mathbf{c}}(L)=\underline{\mathbf{b}}(L) \mathbf{Q}$. Because $\mathbf{v}_{t}$ is fundamental for $\mathbf{y}_{s t}, \mathbf{b}_{s}(0)$ has rank $q$, see (II), so that $\underline{\mathbf{b}}(0)=\underline{\mathbf{b}}_{0}$ has $\operatorname{rank} q$.

Q.E.D.

Summing up, given the infinite-dimensional vector $\mathbf{y}_{t}$, assuming A.1, finite rank, rational spectral density, and the existence of a one-sided rational moving average representation, we obtain the existence of a rational fundamental representation for $\mathbf{y}_{t}$, which is unique up to multiplication by an orthogonal matrix. Moreover, for some $s$, the space spanned by the current and past values of $\mathbf{y}_{s t}$ coincides with the space spanned by current and past values of the whole vector $\mathbf{y}_{t}$ (equivalently, a fundamental white noise of $\mathbf{y}_{s t}$ is a fundamental white noise of $\mathbf{y}_{t}$ ).

Let us now return to the infinite-dimensional vector $\mathbf{x}_{t}$ and to the decomposition $\mathbf{x}_{t}=\chi_{t}+\boldsymbol{\xi}_{t}$. Assume that $\chi_{t}$ has rational spectral density, so that either $\operatorname{rank}\left(\boldsymbol{\Sigma}_{n}^{\chi}(\theta)\right)<$ $q$ for all $\theta \in[-\pi \pi]$ or $\operatorname{rank}\left(\Sigma_{n}^{\chi}(\theta)\right)=q$ for almost all $\theta$ in $[-\pi \pi]$. On the other hand, since $\lambda_{n q}^{\chi}(\theta)$ diverges for almost all $\theta$ in $[-\pi \pi]$, this is Assumption A.2, there exists $s$ such that $\operatorname{rank}\left(\Sigma_{n}^{\chi}(\theta)\right)=q$ for $n \geq s$ and almost all $\theta$ in $[-\pi \pi]$. Therefore, $\chi_{t}$ has $\operatorname{rank} q$.

Adding to a rational spectral density the assumption that $\chi_{t}$ has a one-sided rational representation or, equivalently, that $\mathcal{H}_{t}^{\chi s}=\mathcal{H}_{t}^{\chi}$ for some $s$, so that cases like (3.1) cannot occur, Lemma 2 ensures that $\chi_{t}$ has a rational fundamental representation. 
More precisely, for $i \in \mathbb{N}$,

$$
\chi_{i t}=\frac{c_{i 1}(L)}{d_{i 1}(L)} u_{1 t}+\frac{c_{i 2}(L)}{d_{i 2}(L)} u_{2 t}+\cdots+\frac{c_{i q}(L)}{d_{i q}(L)} u_{q t}
$$

where $c_{i f}(L)$ and $d_{i f}(L)$ are polynomials in $L$, and $\mathbf{u}_{t}$ is fundamental for $\chi_{t}$.

However, in Assumption A.3 (see Section 4.2), we will require more than the existence of an integer $s$ such that $\mathcal{H}_{t}^{\chi_{s}}=\mathcal{H}_{t}^{\chi}$. Rather, we suppose that the space spanned by $\chi_{i_{1} \tau}, \chi_{i_{2} \tau}, \ldots, \chi_{i_{q+1}, \tau}, \tau \leq t$, coincides with $\mathcal{H}_{t}^{\chi}$ for all $(q+1)$-tuples $i_{1}<i_{2}<\cdots<$ $i_{q+1}$. Thus, $\mathbf{u}_{t}$ in (3.5) is fundamental for any $(q+1)$-dimensional subvector of $\chi_{t}$, not only for the subvector $\chi_{s t}$ associated with some $s$. This stronger requirement is motivated in Section 4. We prove that, under a quite general parameterization, the stronger condition holds generically, i.e. outside of a negligible subset, as defined in Section 4 , of the parameter space.

\section{AR representations of the vector $\chi_{t}$}

\subsection{General results for singular stochastic vectors}

Consider an $n$-dimensional vector $\mathbf{y}_{t}$ such that

$$
y_{i t}=\frac{c_{i 1}(L)}{d_{i 1}(L)} v_{1 t}+\frac{c_{i 2}(L)}{d_{i 2}(L)} v_{2 t}+\cdots+\frac{c_{i q}(L)}{d_{i q}(L)} v_{q t}
$$

with

$$
c_{i f}(L)=c_{i f, 0}+c_{i f, 1} L+\cdots+c_{i f, s_{1}} L^{s_{1}} \quad \text { and } \quad d_{i f}(L)=1+d_{i f, 1} L+\cdots+d_{i f, s_{2}} L^{s_{2}}
$$

for $i=1,2, \ldots, n, f=1,2, \ldots, q$, where $\mathbf{v}_{t}=\left(v_{1 t} v_{2 t} \cdots v_{q t}\right)$ is orthonormal white noise.

We assume that for any $i$ the filters in (4.2) are parameterized in the same set $\Pi \subset \mathbb{R}^{\nu}$, with $\nu=q\left(s_{1}+s_{2}+1\right)$, where

(I) $\Pi$ is the closure of an open subset of $\mathbb{R}^{\nu}$;

(II) $d_{i f}(L)$ has no root of modulus smaller than or equal to one, for $f=1,2, \ldots, q$.

Thus, there exists a real $\phi>1$ such that all the roots of the polynomials $d_{i f}(L)$ are of modulus greater than or equal to $\phi$. 
As a consequence, the vector $\mathbf{y}_{t}$ is described by a parameter vector taking values in $\Pi^{n}=\underbrace{\Pi \times \Pi \times \cdots \times \Pi}_{n}$, which is the closure of a non-empty open subset of $\mathbb{R}^{\mu}$, with $\mu=n \nu$.

We are interested in the case $n>q$. Such "tall systems" have been studied recently by Anderson, Deistler and their coauthors (see in particular, Anderson and Deistler, 2008a and b). One of their results is that when $n>q$, there exists a nowhere dense set $\mathcal{N} \subset \Pi^{n}$, i.e. a set whose closure has no interior points, such that if the parameter vector lies in $\Pi^{n}-\mathcal{N}, \mathbf{y}_{t}$ has an autoregressive representation of the form

$$
\mathbf{A}(L) \mathbf{y}_{t}=\mathbf{R} \mathbf{v}_{t}
$$

where

(i) $\mathbf{R}$ is $n \times q$, with $\operatorname{rank}(\mathbf{R})=q$;

(ii) $\mathbf{A}(L)$ is an $n \times n$ finite-degree matrix polynomial.

When a property holds in $\Pi^{n}-\mathcal{M}$ and $\mathcal{M}$ is nowhere dense in $\Pi^{n}$, we say that the property holds generically in $\Pi^{n}$. As $\mathbf{R}$ has generically full rank, (4.3) implies that, generically, $\mathbf{v}_{t}$ is fundamental for $\mathbf{y}_{t}{ }^{9}$

To provide an intuition for this result and Proposition 1 below, let us consider the following elementary example, in which $n=2, q=1$, and

$$
\begin{aligned}
& y_{1 t}=a_{1} v_{t}+b_{1} v_{t-1} \\
& y_{2 t}=a_{2} v_{t}+b_{2} v_{t-1},
\end{aligned}
$$

with parameter $\left(a_{1}, b_{1}, a_{2}, b_{2}\right)$ in $\mathbb{R}^{2} \times \mathbb{R}^{2}$. Outside of the nowhere dense subset in which $a_{1} b_{2}-a_{2} b_{1}=0$, we obtain

$$
v_{t}=\frac{1}{a_{1} b_{2}-a_{2} b_{1}}\left(b_{2} y_{1 t}-b_{1} y_{2 t}\right) .
$$

Using (4.5) to get rid of $v_{t-1}$ in (4.4), we obtain the $\mathrm{AR}(1)$ representation

$$
\begin{aligned}
& y_{1 t}=d b_{1} b_{2} y_{1 t-1}-d b_{1}^{2} y_{2 t-1}+a_{1} v_{t} \\
& y_{2 t}=d b_{2}^{2} y_{1 t-1}-d b_{1} b_{2} y_{2 t-1}+a_{2} v_{t},
\end{aligned}
$$

where $d=1 /\left(a_{1} b_{2}-a_{2} b_{1}\right)$. Note that

\footnotetext{
${ }^{9}$ Results on the existence of autoregressive representations for singular vectors are given in Miamee and Pourahmadi (1987). Without assuming rational spectral density, they provide sufficient conditions. However, the existence of finite-degree autoregressive representation is not considered.
} 
(i) If $a_{1} b_{2}-a_{2} b_{1}=0$, no finite-degree autoregressive representation exists, unless $b_{1}=$ $b_{2}=0$. Moreover, fundamentalness of $v_{t}$ for $\mathbf{y}_{t}$ requires that the root of $a_{1}+b_{1} L$ (which is also the root of $a_{2}+b_{2} L$ ) has modulus larger than one.

(ii) However, as soon as $a_{1} b_{2}-a_{2} b_{1} \neq 0, v_{t}$ is fundamental for $\mathbf{y}_{t}$ even if both the roots of $a_{i}+b_{i} L, i=1,2$, are smaller than one in modulus.

(iii) Quite obviously, $a_{1} b_{2}-a_{2} b_{1} \neq 0$ if and only if $y_{1 t-1}$ and $y_{2 t-1}$ are linearly independent. Therefore, generically, the projection (4.6) is unique, i.e. generically no other autoregressive representation of degree one exists.

(iv) But higher-degree autoregressive representations do exist. Rewriting (with obvious definitions of $\mathbf{A}$ and $\mathbf{a}$ ) (4.6) as $\mathbf{y}_{t}=\mathbf{A} \mathbf{y}_{t-1}+\mathbf{a} v_{t}$, we get $\mathbf{y}_{t}=\mathbf{A}^{2} \mathbf{y}_{t-2}+$ $\mathbf{A} \mathbf{a} v_{t-1}+\mathbf{a} v_{t}$. Using (4.5) to get rid of $v_{t-1}$, we obtain another autoregressive representation, of degree two. Such non-uniqueness does not occur for square systems (when $n=q$ ).

(v) On the other hand, if $n=3$ and $y_{i t}=a_{i} v_{t}+b_{i} v_{t-1}, i=1,2,3$, then, outside of the set in which $a_{2} b_{1}=a_{1} b_{2}$ and $a_{3} b_{1}=a_{1} b_{3}$, which is nowhere dense in $\mathbb{R}^{2} \times \mathbb{R}^{2} \times \mathbb{R}^{2}$, we have

$$
v_{t}=\frac{1}{a_{1} \gamma_{1}+a_{2} \gamma_{2}+a_{3} \gamma_{3}}\left(\gamma_{1} y_{1 t}+\gamma_{2} y_{2 t}+\gamma_{3} y_{3 t}\right)
$$

where $b_{1} \gamma_{1}+b_{2} \gamma_{2}+b_{3} \gamma_{3}=0$. This can be used to get rid of $v_{t-1}$, in the same way as we did in the $n=2$ case. Thus, generically, $\mathbf{y}_{t}$ has an $\operatorname{AR}(1)$ representation. However, the variables $y_{i t-1}, i=1,2,3$, are not linearly independent, so that such minimum-lag autoregressive representation is not unique.

Let us show that remark (iii) can be generalized. Precisely, if $n=q+1$, then, generically, there exists only one minimal-lag autoregressive representation.

Proposition 1 Consider an $n$-dimensional vector $\mathbf{y}_{t}$ with representation (4.1)-(4.2), and assume that $n=q+1$. There exists a set $\mathcal{N} \subset \Pi^{q+1}$, nowhere dense in $\Pi^{q+1}$, such that, if the parameter vector lies in $\Pi^{q+1}-\mathcal{N}$,

(i) $\mathbf{y}_{t}$ has a finite-degree $A R$ representation $\mathbf{A}(L) \mathbf{y}_{t}=\mathbf{R v}_{t}$, where $\mathbf{R}$ is $(q+1) \times q$, $R_{i f}=c_{i f}(0), \operatorname{rank}(\mathbf{R})=q, \mathbf{A}(L)$ is $(q+1) \times(q+1)$ and has degree not exceeding $S=q s_{1}+q^{2} s_{2}$. This implies that $\mathbf{v}_{t}$ is fundamental for $\mathbf{y}_{t}$. 
(ii) Suppose that $(a) \mathbf{A}^{*}(L)$ is a $(q+1) \times(q+1)$ polynomial matrix whose degree does not exceed $S$, with $\mathbf{A}^{*}(0)=\mathbf{I}$, (b) $\mathbf{R}^{*}$ is $(q+1) \times q$, (c) $\mathbf{v}_{t}^{*}$ is a q-dimensional orthonormal white noise orthogonal to $\mathbf{y}_{t-k}, k \geq 1$, (d) $\mathbf{A}^{*}(L) \mathbf{y}_{t}=\mathbf{R}^{*} \mathbf{v}_{t}^{*}$. Then $\mathbf{A}^{*}(L)=\mathbf{A}(L), \mathbf{R}^{*}=\mathbf{R} \mathbf{Q}, \mathbf{v}_{t}^{*}=\mathbf{Q}^{\prime} \mathbf{v}_{t}$, where $\mathbf{Q}$ is an orthogonal $q \times q$ matrix.

See Appendix A for the proof.

Part (i) of Proposition 1 has already been proved in the papers by Anderson and Deistler, as we have mentioned above. However, the parameters in Anderson and Deistler's papers are the entries of the matrices in the state-space representation of the rational-spectrum vector $\mathbf{y}_{t}$, whereas our parameters are the coefficients of the rational functions in representation (4.1).

Note that Proposition 1 does not claim that, generically, the process $\mathbf{y}_{t}$ corresponding to a parameter vector in $\Pi^{q+1}$ has no non-fundamental representations. What it claims is that, generically, such non-fundamental representations are not parameterized in $\Pi^{q+1}$. For example, representation (4.4) is generically fundamental in $\mathbb{R}^{2} \times \mathbb{R}^{2}$. On the other hand, given any $a$ with $|a|>1$, the process $\mathbf{y}_{t}$ also has the representation

$$
y_{i t}=\left[\left(a_{i}+b_{i} L\right) \frac{1-a L}{1-a^{-1} L}\right]\left[\frac{1-a^{-1} L}{1-a L} v_{t}\right]=\frac{\left(a_{i}+b_{i} L\right)(1-a L)}{1-a^{-1} L} w_{t}
$$

for $i=1,2$, where

$$
w_{t}=\frac{1-a^{-1} L}{1-a L} v_{t}=-a^{-1} F \frac{1-a^{-1} L}{1-a^{-1} F} v_{t}
$$

is white noise (this is easily proved by showing that its spectral density is constant). Thus, $\mathbf{y}_{t}$ has the non-fundamental representation (4.7). The latter, however, is parameterized in $\mathbb{R}^{2} \times \mathbb{R}^{2} \times \mathbb{R}$, not $\mathbb{R}^{2} \times \mathbb{R}^{2}$.

Now assume that $\mathbf{y}_{t}$ is infinite-dimensional with $y_{i t}$ modeled as in (4.1) for $i \in \mathbb{N}$. The vector $\mathbf{y}_{t}$ is parameterized in $\Pi^{\infty}=\Pi \times \Pi \times \cdots$. We define negligible sets and genericity in $\Pi^{\infty}$ with respect to the product topology ${ }^{10}$. We say that a subset of $\Pi^{\infty}$ is negligible if it is meagre, i.e. the union of a countable set of nowhere dense subsets, and that a property holds generically in $\Pi^{\infty}$ if the subset where it does not hold is meagre.

\footnotetext{
${ }^{10}$ Let us recall that a basis for the open sets in $\Pi^{\infty}$ in the product topology is the family of all sets $\prod_{i=1}^{\infty} G_{i}$, where $G_{i}$ is an open subset of $\Pi$ and $G_{i}=\Pi$ but for a finite number of values of $i$.
} 
Define the set $\mathcal{M}_{m}$, for $m \geq q+1$, as the set of points in $\Pi^{\infty}$ such that all vectors $\mathbf{y}_{t}^{i_{1}, i_{2}, \ldots, i_{q+1}}=\left(y_{i_{1} t} y_{i_{2} t} \cdots y_{i_{q+1} t}\right)$, with $i_{1}<i_{2}<\cdots<i_{q+1} \leq m$, admit a representation of the form

$$
\mathbf{A}^{i_{1}, i_{2}, \ldots, i_{q+1}}(L) \mathbf{y}_{t}^{i_{1}, i_{2}, \ldots, i_{q+1}}=\mathbf{R}^{i_{1}, i_{2}, \ldots, i_{q+1}} \mathbf{v}_{t}
$$

where $\mathbf{A}^{i_{1}, i_{2}, \ldots, i_{q+1}}(L)$ is at most of degree $S$ and unique in the sense of Proposition $1(\mathrm{~b})$. From Proposition 1, we see that $\mathcal{N}_{m}=\Pi^{\infty}-\mathcal{M}_{m}$ is a nowhere dense subset in the product topology of $\Pi^{\infty}$, so that the set $\mathcal{N}=\cup_{m=q+1}^{\infty} \mathcal{N}_{m}$, being a countable union of nowhere dense subsets of $\Pi^{\infty}$, is a meagre subset. We thus have the following.

Lemma 3 Assume that $\mathbf{y}_{t}$ is infinite-dimensional, modeled as in (4.1) for $i \in \mathbb{N}$ and parameterized in $\Pi^{\infty}$. Generically in $\Pi^{\infty}$, all the vectors $\mathbf{y}_{t}^{i_{1}, i_{2}, \ldots, i_{q+1}}=\left(y_{i_{1} t} y_{i_{2} t} \cdots y_{i_{q+1} t}\right)$, with $i_{1}<i_{2}<\cdots<i_{q+1}$, can be represented as in (4.8), where $\mathbf{A}^{i_{1}, i_{2}, \ldots, i_{q+1}}(L)$ is at most of degree less than $S$ and unique in the sense of Proposition 1(b).

Definining negligible subsets of $\Pi^{\infty}$ as meagre subsets has a good motivation in the fact that (i) the complement of a meagre subset of $\Pi^{\infty}$ is not meagre, (ii) if a subset of $\Pi^{\infty}$ is not meagre, obtaining it as the union of a family of nowhere dense subsets requires an uncountable family. ${ }^{11}$

Moreover, assuming that the parameter space indexing the polynomials $c_{i j}(L)$ and $d_{i j}(L)$ does not depend on $i$, as we do in (4.1), is convenient but not necessary. With the dimension of the parameter space depending on $i$, a more general version of Proposition 1 holds as well as the meagreness result for infinite-dimensional vectors $\mathbf{y}_{t}$. However, the gain in generality does not seem to justify the substantial additional complications in the proof of Proposition 1 and the determination of the degree of $\mathbf{A}(L)$.

\footnotetext{
${ }^{11}$ Let us recall that: (I) because $\Pi$ is a closed subset of $\mathbb{R}^{\nu}$, the space $\Pi^{\infty}$ is the Cartesian product of a countable family of complete metric spaces and is therefore a complete metric space (Dunford and Schwartz (1988), p. 32, Lemma 4); (II) in complete metric spaces the complement of a meagre subset is not meagre (same reference, Baire Category Theorem, p. 20).
} 


\subsection{Existence of AR representations of $\chi_{t}$}

Let us now turn our attention to the common-component vector $\chi_{t}$. As we have seen, assuming that $\chi_{t}$ has rational spectral density and a one-sided rational representation implies, by Lemma 2 , that $\chi_{t}$ has a fundamental rational representation of the form (4.1). The meagreness argument developed in Section 4.1, as summarized in Lemma 3, provides a motivation for assuming more.

Assumption A.3 The vector $\chi_{t}$ has a representation

where

$$
\chi_{i t}=\frac{c_{i 1}(L)}{d_{i 1}(L)} u_{1 t}+\frac{c_{i 2}(L)}{d_{i 2}(L)} u_{2 t}+\cdots+\frac{c_{i q}(L)}{d_{i q}(L)} u_{q t},
$$

$$
c_{i f}(L)=c_{i f, 0}+c_{i f, 1} L+\cdots+c_{i f, s_{1}} L^{s_{1}} \quad \text { and } \quad d_{i f}(L)=1+d_{i f, 1} L+\cdots+d_{i f, s_{2}} L^{s_{2}}
$$

for all $i \in \mathbb{N}$ and $f=1,2, \ldots, q$. Moreover,

(i) Each vector $\chi_{t}^{i_{1}, i_{2}, \ldots, i_{q+1}}=\left(\chi_{i_{1} t} \chi_{i_{2} t} \cdots \chi_{i_{q+1} t}\right)^{\prime}$, with $i_{1}<i_{2}<\cdots<i_{q+1}$, has an autoregressive representation

$$
\mathbf{A}^{i_{1}, i_{2}, \ldots, i_{q+1}}(L) \chi_{t}^{i_{1}, i_{2}, \ldots, i_{q+1}}=\mathbf{R}^{i_{1}, i_{2}, \ldots, i_{q+1}} \mathbf{u}_{t}
$$

where $\mathbf{A}^{i_{1}, i_{2}, \ldots, i_{q+1}}(L)$ is of degree not greater than $S=q s_{1}+q^{2} s_{2}$, and $\mathbf{R}^{i_{1}, i_{2}, \ldots, i_{q+1}}$ has rank $q$. This implies that $\mathbf{u}_{t}$ is fundamental for all $(q+1)$-dimensional subvectors of $\chi_{t}$.

(ii) Representation (4.9) is unique in the sense of Proposition 1(ii).

An immediate consequence of Assumption A.3 is that $\chi_{t}$ can be represented as in (1.8), that is,

$$
\mathbf{A}^{1}(L)\left(\begin{array}{c}
\chi_{1 t} \\
\chi_{2 t} \\
\vdots \\
\chi_{q+1, t}
\end{array}\right)=\mathbf{R}^{1} \mathbf{u}_{t}, \quad \mathbf{A}^{2}(L)\left(\begin{array}{c}
\chi_{q+2, t} \\
\chi_{q+3, t} \\
\vdots \\
\chi_{2(q+1), t}
\end{array}\right)=\mathbf{R}^{2} \mathbf{u}_{t}, \quad \ldots
$$

where the degrees of the polynomial matrices $\mathbf{A}^{k}(L)$ do not exceed $S$. Moreover, those $\mathbf{A}^{k}(L)$ 's are unique among autoregressive representations of degree not greater 
than $S$. Writing $\underline{\mathbf{A}}(L)$ for the (infinite) block-diagonal matrix with diagonal blocks $\mathbf{A}^{1}(L), \mathbf{A}^{2}(L), \ldots$, and letting $\underline{\mathbf{R}}=\left(\mathbf{R}^{1 \prime}, \mathbf{R}^{2 \prime}, \cdots\right)^{\prime}$, we thus have

$$
\underline{\mathbf{A}}(L) \chi_{t}=\underline{\mathbf{R}} \mathbf{u}_{t}
$$

Two comments are in order. Firstly, of course, any permutation of the variables produces a distinct $(q+1)$-blockwise autoregressive representation of the form (4.10). This is consistent with the observation in Section 4.1 that autoregressive representations of singular vectors are not unique, even if their degree is minimum, unless $n=q+1$, see Proposition 1.

Secondly, $\mathbf{u}_{t}$ and $\underline{\mathbf{R}}$ do not play any special role. By Lemma 2(ii), all the white noise vectors $\tilde{\mathbf{u}}_{t}$ and matrices $\tilde{\mathbf{R}}$, corresponding to alternative representations of the form (4.11) satisfy $\tilde{\mathbf{R}}=\mathbf{Q R}$, and $\tilde{\mathbf{u}}_{t}=\mathbf{Q}^{\prime} \mathbf{u}_{t}$ where $\mathbf{Q}$ is an orthogonal $q \times q$ matrix. ${ }^{12}$ For identification and estimation of a couple $\mathbf{u}_{t}^{*}, \underline{\mathbf{R}}^{*}$ based on economic theory, see Forni et al. (2009) and Forni et al. (2014).

\subsection{Construction of the AR representations of $\chi_{t}$}

Assumption A.3 ensures existence and uniqueness of the autoregressive representation (4.10). We now show how (4.10), i.e. the matrices $\mathbf{A}^{k}(L)$ and (up to multiplication by an orthogonal matrix) $\mathbf{R}^{k}$, can be constructed from the spectral density of the $\chi$ 's.

(i) Assume that the population spectral density of the vector $\chi_{t}$ is known, i.e. that the nested spectral density matrices $\Sigma_{n}^{\chi}(\theta), n \in \mathbb{N}$, are known.

(ii) Denote by $\chi_{t}^{k}$ the $k$-th $(q+1)$-dimensional subvector of $\chi_{t}$ appearing in (4.10), and write $\boldsymbol{\Sigma}_{j k}^{\chi}(\theta)$ for the $(q+1) \times(q+1)$ cross-spectral density between $\chi_{t}^{j}$ and $\chi_{t}^{k}$. Then, denoting by $\Gamma_{j k, s}^{\chi}$ the covariance between $\chi_{t}^{j}$ and $\chi_{t-s}^{k}$,

$$
\Gamma_{j k, s}^{\chi}=\mathrm{E}\left[\chi_{t}^{j} \chi_{t-s}^{k}{ }^{\prime}\right]=\int_{-\pi}^{\pi} e^{i s \theta} \Sigma_{j k}^{\chi}(\theta) d \theta
$$

(iii) Using the autocovariance function $\Gamma_{k k, s}^{\chi}$, we obtain the minimum-lag matrix polynomial $\mathbf{A}^{k}(L)$ and the autocovariance function of the unobservable vectors

$$
\boldsymbol{\Psi}_{t}^{1}=\mathbf{A}^{1}(L) \chi_{t}^{1}, \quad \Psi_{t}^{2}=\mathbf{A}^{2}(L) \chi_{t}^{2}, \quad \ldots
$$

\footnotetext{
${ }^{12}$ Of course, $\underline{\mathbf{R}} \mathbf{u}_{t}$, which is the one-step-ahead prediction error of $\boldsymbol{\chi}_{t}$, is identified.
} 
Indeed, letting $\mathbf{A}^{k}(L)=\mathbf{I}_{q+1}-\mathbf{A}_{1}^{k} L-\cdots-\mathbf{A}_{S}^{k} L^{S}$, define

$$
\mathbf{A}^{[k]}=\left(\begin{array}{llll}
\mathbf{A}_{1}^{k} & \mathbf{A}_{2}^{k} & \cdots & \mathbf{A}_{S}^{k}
\end{array}\right), \quad \mathbf{B}_{k}^{\chi}=\left(\boldsymbol{\Gamma}_{k k, 1}^{\chi} \Gamma_{k k, 2}^{\chi} \cdots \Gamma_{k k, S}^{\chi}\right)
$$

and

$$
\mathbf{C}_{j k}^{\chi}=\left(\begin{array}{cccc}
\Gamma_{j k, 0}^{\chi} & \Gamma_{j k, 1}^{\chi} & \cdots & \Gamma_{j k, S-1}^{\chi} \\
\Gamma_{j k,-1}^{\chi} & \Gamma_{j k, 0}^{\chi} & \cdots & \Gamma_{j k, S-2}^{\chi} \\
\vdots & & & \vdots \\
\Gamma_{j k,-S+1}^{\chi} & \Gamma_{j k,-S+2}^{\chi} & \cdots & \Gamma_{j k, 0}^{\chi}
\end{array}\right)
$$

We have

$$
\mathbf{A}^{[k]}=\mathbf{B}_{k}^{\chi}\left(\mathbf{C}_{k k}^{\chi}\right)^{-1}=\mathbf{B}_{k}^{\chi}\left(\mathbf{C}_{k k}^{\chi}\right)_{\mathrm{ad}} \operatorname{det}\left(\mathbf{C}_{k k}^{\chi}\right)^{-1} \text { and } \quad \Gamma_{j k}^{\psi}=\Gamma_{j k}^{\chi}-\mathbf{A}^{[j]} \mathbf{C}_{j k}^{\chi} \mathbf{A}^{[k]},
$$

where $\mathbf{C}_{\text {ad }}$ stands for the adjoint of a square matrix $\mathbf{C}$. Invertibility of $\mathbf{C}_{k k}^{\chi}$, hence of $\left(\mathbf{C}_{k k}^{\chi}\right)_{\mathrm{ad}}$, is a consequence of Assumption A.3.

(iv) The $\infty \times \infty$ matrix $\underline{\Gamma}^{\Psi}$ obtained by piecing together the matrices $\Gamma_{j k}^{\Psi}$ is of rank $q$ (see Lemma 2(iii)) and can therefore be represented as $\underline{\Gamma}^{\Psi}=\underline{\mathbf{S}} \underline{\mathbf{S}}^{\prime}$, where $\underline{\mathbf{S}}$ is an $\infty \times q$ matrix. On the other hand, $\underline{\Gamma}^{\Psi}$ is the covariance matrix of the right-hand side terms in (4.10), so that $\underline{\mathbf{S}}=\underline{\mathbf{R}} \mathbf{H}$, where $\mathbf{H}$ is $q \times q$ and orthogonal.

Lastly, using $\mathbf{x}_{t}=\chi_{t}+\boldsymbol{\xi}_{t}$, letting $\mathbf{Z}_{t}=\underline{\mathbf{A}}(L) \mathbf{x}_{t}$ and $\boldsymbol{\Phi}_{t}=\underline{\mathbf{A}}(L) \boldsymbol{\xi}_{t}$, we obtain

$$
\mathbf{Z}_{t}=\underline{\mathbf{R}} \mathbf{u}_{t}+\Phi_{t}
$$

In conclusion, starting with the spectral density of the $\chi$ 's, we obtain the filter $\underline{\mathbf{A}}(L)$, the vector $\mathbf{Z}_{t}$ and the model (4.17). The above construction, based on an estimate of the spectral density $\Sigma_{n}^{\chi}(\theta)$, is used in the estimation procedure studied in Forni et al. (2014), see Section 4.4 for an illustration.

\subsection{Normalization of $\mathrm{Z}_{t}$}

Under our assumptions, the dynamic factor model for the variables $x_{i t}$ has been transformed into model (4.17), which has the form (2.2) for the variables $Z_{i t}$, with $r=q$ and $\mathbf{F}_{t}=\mathbf{u}_{t}$. Application of standard principal components to estimate $\mathbf{u}_{t}$ and $\underline{\mathbf{R}}$ 
requires that Assumptions B.1 and B.2 be fulfilled. The latter are equivalent to statements (i), (ii) and (iii) of Theorem B, see Section 2.2. In particular, the first eigenvalue of the variance-covariance matrix of $\boldsymbol{\Phi}_{n t}$ should be bounded. We show below that this is not a consequence of our assumptions so far.

To see this, let us resort again to the simple case in which $q=1$ and the common components are MA(1),

$$
x_{i t}=u_{t}+c_{i} u_{t-1}+\xi_{i t}
$$

Considering the 2-dimensional vectors $\chi_{t}^{k}$, we have, see (4.6):

$$
\mathbf{A}^{k}(L)=\mathbf{I}_{2}-\left(c_{k}-c_{k-1}\right)^{-1}\left(\begin{array}{cc}
c_{k-1} c_{k} & -c_{k-1}^{2} \\
c_{k}^{2} & -c_{k-1} c_{k}
\end{array}\right) L
$$

Assumption 3 implies that $c_{k}-c_{k-1} \neq 0$ for all $k$ (and all possible groupings), but no more. In particular, it does not imply that $\left|c_{k}-c_{k-1}\right| \geq d$ for some $d>0$ and all $k$. As a consequence, the variance of the components of $\boldsymbol{\Phi}_{t}=\underline{\mathbf{A}}(L) \boldsymbol{\xi}_{t}$ is not necessarily bounded, as it should be if $\boldsymbol{\Phi}_{t}$ were idiosyncratic.

Two possible ways out of this difficulty are: (i) assuming that $\operatorname{det}\left(\mathbf{C}_{k k}^{\chi}\right) \geq c>0$ for all $k$, this is what we do in our companion paper on estimation, Forni et al. (2014), (ii) normalizing $\mathbf{Z}_{t}$, this is what we do here. Define:

(i) $w_{i}=1$ if $\operatorname{var}\left(Z_{i t}\right)=0$, otherwise $w_{i}=\sqrt{\operatorname{var}\left(Z_{i t}\right)}$;

(ii) $\underline{\mathbf{V}}$ as the $\infty \times \infty$ diagonal matrix with $w_{i}^{-1}$ in entry $(i, i)$;

(iii) $\mathbf{z}_{t}=\underline{\mathbf{V}} \mathbf{Z}_{t}, \underline{\mathbf{r}}=\underline{\mathbf{V}} \underline{\mathbf{R}}, \phi_{t}=\underline{\mathbf{V}} \boldsymbol{\Phi}_{t}$

Equation (4.17) becomes

$$
\mathbf{z}_{t}=\underline{\mathbf{r}} \mathbf{u}_{t}+\phi_{t}
$$

Adding the following assumption is sufficient, though not necessary, to prove that $\boldsymbol{\phi}_{t}$ fulfills statement (i) of Theorem B.

Assumption A.4 There exists a real $b^{\xi}>0$ such that $\lambda_{n n}^{\xi}(\theta) \geq b^{\xi}$ for all $n$ and $\theta$ almost everywhere in $[-\pi \pi]\left(\lambda_{n n}^{\xi}(\theta)\right.$ is the smallest eigenvalue of $\Sigma_{n}^{\xi}(\theta)$, see Section 2.2). 
Proposition 2 Let $\boldsymbol{\Gamma}_{n}^{\phi}$ the variance-covariance matrix of $\phi_{n t}$ and $\mu_{n 1}^{\phi}$ its first eigenvalue. Under Assumptions A.1, A.2, A.3 and A.4, there exists a real number $M$ such that $\mu_{n 1}^{\phi} \leq M$ for all $n$.

Proof. It is convenient here to assume, without loss of generality, that the number $n$ of variables increases by blocks of size $q+1$. Thus $n=m(q+1)$, where $m$ is the number of blocks. Let $\mathbf{b}$ be a $1 \times n$ vector with $|\mathbf{b}|=1$. The notation $\mathbf{b}=\left(\mathbf{b}^{1} \mathbf{b}^{2} \cdots \mathbf{b}^{m}\right)$ and $\mathbf{V}_{m}=\operatorname{diag}\left(\mathbf{V}^{1} \mathbf{V}^{2} \ldots \mathbf{V}^{m}\right)$ is used in an obvious way. We denote by $\Sigma^{\xi k}(\theta)$ the spectral density matrix of $\boldsymbol{\xi}_{t}^{k}$ and by $\mathbf{a}_{j}^{k}\left(e^{-i \theta}\right)$ the $j$-th row of $\mathbf{A}^{k}\left(e^{-i \theta}\right)$, for $j=1,2, \ldots, q+1$. Let $\mathbf{c}=\left(\mathbf{c}^{1} \mathbf{c}^{2} \cdots \mathbf{c}^{m}\right)$, and suppose that $\mathbf{c}^{j}=\mathbf{0}$ if $j \neq k$. Then $\mathbf{c} \boldsymbol{\Sigma}_{n}^{\xi}(\theta) \mathbf{c}^{\prime}=\mathbf{c}^{k} \boldsymbol{\Sigma}^{\xi k}(\theta) \mathbf{c}^{k^{\prime}}$. As a consequence, if $\mathbf{d}$ is $1 \times(q+1)$, then

$$
\lambda_{n n}^{\xi}(\theta) \mathbf{d d}^{\prime} \leq \mathbf{d} \boldsymbol{\Sigma}^{\xi k}(\theta) \mathbf{d}^{\prime} \leq \lambda_{n 1}^{\xi}(\theta) \mathbf{d d}^{\prime},
$$

for $k=1,2, \ldots, m$. Using Assumption A.4, statement (i) of Theorem A and (4.19),

$$
\begin{aligned}
& \mathbf{b} \boldsymbol{\Sigma}_{n}^{\phi}(\theta) \mathbf{b}^{\prime}=\mathbf{b V A}\left(e^{-i \theta}\right) \mathbf{\Sigma}^{\xi}(\theta) \mathbf{A}^{\prime}\left(e^{i \theta}\right) \mathbf{V} \mathbf{b}^{\prime} \leq \lambda_{n 1}^{\xi}(\theta) \mathbf{b V A}\left(e^{-i \theta}\right) \mathbf{A}^{\prime}\left(e^{i \theta}\right) \mathbf{V} \mathbf{b}^{\prime} \\
&=\lambda_{n 1}^{\xi}(\theta) \sum_{k=1}^{m} \mathbf{b}^{k} \mathbf{V}^{k} \mathbf{A}^{k}\left(e^{-i \theta}\right) \mathbf{A}^{k^{\prime}}\left(e^{i \theta}\right) \mathbf{V}^{k} \mathbf{b}^{k^{\prime}} \\
& \leq \lambda_{n 1}^{\xi}(\theta) \sum_{k=1}^{m} \mathbf{b}^{k} \operatorname{trace}\left[\mathbf{V}^{k} \mathbf{A}^{k}\left(e^{-i \theta}\right) \mathbf{A}^{k^{\prime}}\left(e^{i \theta}\right) \mathbf{V}^{k}\right] \mathbf{b}^{k^{\prime}}=\lambda_{n 1}^{\xi}(\theta) \sum_{k=1}^{m} \mathbf{b}^{k}\left[\sum_{j=1}^{q+1} \frac{\mathbf{a}_{j}^{k}\left(e^{-i \theta}\right) \mathbf{a}_{j}^{k^{\prime}}\left(e^{i \theta}\right)}{\operatorname{var}\left(Z_{i t}^{k}\right)}\right] \mathbf{b}^{k^{\prime}} \\
& \leq \lambda_{n 1}^{\xi}(\theta) \sum_{k=1}^{m} \mathbf{b}^{k}\left[\sum_{j=1}^{q+1} \frac{\mathbf{a}_{j}^{k}\left(e^{-i \theta}\right) \mathbf{a}_{j}^{k^{\prime}}\left(e^{i \theta}\right)}{\int_{-\pi}^{\pi} \mathbf{a}_{j}^{k}\left(e^{-i \theta}\right) \Sigma^{\xi k}(\theta) \mathbf{a}_{j}^{k^{\prime}}\left(e^{i \theta}\right) d \theta}\right] \mathbf{b}^{k^{\prime}} \\
& \leq \frac{B^{\xi}}{b^{\xi}} \sum_{k=1}^{m} \mathbf{b}^{k}\left[\sum_{j=1}^{q+1} \frac{\mathbf{a}_{j}^{k}\left(e^{-i \theta}\right) \mathbf{a}_{j}^{k^{\prime}}\left(e^{i \theta}\right)}{\int_{-\pi}^{\pi} \mathbf{a}_{j}^{k}\left(e^{-i \theta}\right) \mathbf{a}_{j}^{k^{\prime}}\left(e^{-i \theta}\right) d \theta}\right] \mathbf{b}^{k^{\prime}},
\end{aligned}
$$

$\theta$-a.e. in $[-\pi \pi]$. Integrating, we obtain

$$
\mathbf{b} \Gamma_{n}^{\phi} \mathbf{b}^{\prime}=\int_{-\pi}^{\pi} \mathbf{b} \Sigma_{n}^{\phi}(\theta) \mathbf{b}^{\prime} d \theta \leq \frac{B^{\xi}}{b^{\xi}}(q+1),
$$

which implies that $\mu_{n 1}^{\phi}=\max _{|\mathbf{b}|=1} \mathbf{b} \Gamma_{n}^{\phi} \mathbf{b}^{\prime}$ is bounded.

Q.E.D.

Let us now consider statements (ii) and (iii) of Theorem B. The definition of $\phi_{t}$ and statement (i) of Theorem A imply that $\boldsymbol{\phi}_{t}$ and $\boldsymbol{\eta}_{t}=\underline{\mathbf{r}} \mathbf{u}_{t}$ fulfill statement (iii). As 
regards statement (ii), let again $q=1$ and

$$
x_{i t}=\left(c_{i 0}+c_{i 1} L\right) u_{t}+\xi_{i t} .
$$

The corresponding representation (4.18) is

$$
z_{i t}=d_{i 0} u_{t}+\phi_{i t}=\eta_{i t}+\phi_{i t}, \quad d_{i 0}=\frac{c_{i 0}}{\sqrt{c_{i 0}^{2}+\operatorname{var}\left(\Phi_{i t}\right)}} .
$$

We have

$$
\lambda_{n 1}^{\chi}(\theta)=\frac{1}{2 \pi} \sum_{i=1}^{n}\left|c_{i 0}+c_{i 1} e^{-i \theta}\right|^{2} \quad \text { and } \quad \mu_{n 1}^{\eta}=\sum_{i=1}^{n} \frac{c_{i 0}^{2}}{c_{i 0}^{2}+\operatorname{var}\left(\Phi_{i t}\right)} .
$$

We see that divergence of $\lambda_{n 1}^{\chi}(\theta)$ almost everywhere in $[-\pi \pi]$ does not imply divergence of $\mu_{n 1}^{\eta}$. However, convergence of $\mu_{n 1}^{\eta}$ occurs only if $\operatorname{var}\left(\Phi_{i t}\right) / c_{i 0}^{2}$ diverges. Sufficient conditions for this are (1) $\operatorname{var}\left(\Phi_{i t}\right) \rightarrow \infty$ and $c_{i 0}^{2}$ bounded away from zero, $(2) \operatorname{var}\left(\Phi_{i t}\right)$ bounded away from zero and $c_{i 0}^{2} \rightarrow 0$. Regarding (1), though we do not assume that $\operatorname{var}\left(\Phi_{i t}\right)$ is bounded, divergence of $\operatorname{var}\left(\Phi_{i t}\right)$ requires a very special sequence of coefficients $\left(c_{i 0}, c_{i 1}\right)$. Regarding $(2)$, even if we do not assume a positive lower bound for $c_{i 0}$, convergence to zero of $c_{i 0}^{2}$ can be ruled out as very special. Even more farfetched are the cases in which the ratio $\operatorname{var}\left(\Phi_{i t}\right) / c_{i 0}^{2}$ diverges though neither (1) nor (2) holds, like the ratio $\alpha_{1} / \beta_{1}$ with

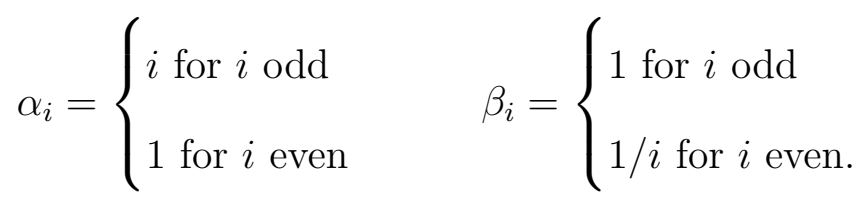

Extending these considerations to $q>1$ and more complex models for $\boldsymbol{\chi}_{t}$ does not seem worthwhile. We believe that the analysis of the simple example above is sufficient to motivate the following assumption on the $q$-th eigenvalue of the variance-covariance matrix of $\mathbf{z}_{t}$.

Assumption A.5 $\mu_{n q}^{\eta} \rightarrow \infty$ as $n \rightarrow \infty$.

Summing up, under Assumptions A.1 through A.5, the variables $x_{i t}$ can be transformed into the variables $z_{i t}$, which satisfy the static model (4.18). Statements (i), (ii) and (iii) of Theorem B hold or, equivalently, the variables $z_{i t}$ fulfill Assumptions B.1 and B.2. 


\subsection{Estimation}

The construction leading from the $x$ 's to the $z$ 's has a natural counterpart in the estimation procedure developed in the companion paper Forni et al. (2014).

(I) We start with an estimate of $\boldsymbol{\Sigma}_{n}^{x}(\theta)$, the spectral density of the observable variables $x_{i t}$; call $\hat{\Sigma}_{n}^{x}(\theta)$ such an estimate.

(II) An estimate of the spectral density of the common components, call it $\hat{\Sigma}_{n}^{\chi}(\theta)$, is then obtained using the first $q$ dynamic principal components of $\hat{\boldsymbol{\Sigma}}_{n}^{x}(\theta)$, see Forni et al. (2000). An estimate of the spectral density of the idiosyncratic components is obtained as well as $\hat{\Sigma}_{n}^{\xi}(\theta)=\hat{\Sigma}_{n}^{x}(\theta)-\hat{\Sigma}_{n}^{\chi}(\theta)$.

(III) Steps (ii), (iii) and (iv) of Section 4.3 are then reproduced, starting with $\hat{\Sigma}_{n}^{\chi}(\theta)$ instead of $\boldsymbol{\Sigma}_{n}^{\chi}(\theta)$. We thus obtain estimates $\hat{\mathbf{A}}^{k}(L), \hat{\mathbf{R}}^{k}, \hat{\mathbf{Z}}_{n t}, \hat{\mathbf{r}}^{k}, \hat{\mathbf{z}}_{n t}$. Note that $\hat{\mathbf{Z}}_{n t}$ and $\hat{\mathbf{z}}_{n t}$ result from the application of one-sided filters to the observable variables $x_{i t}$.

(IV) Lastly, we estimate a static representation with $q$ factors for $\hat{\mathbf{z}}_{n t}$, obtaining an estimate $\hat{\mathbf{u}}_{t}$. This step employs the first $q$ principal components of $\hat{\mathbf{z}}_{n t}$, and therefore only current and past values of the variables $x_{i t}$.

As already observed in the Introduction, though the dynamic model studied in the present paper is more general than model (1.3), when a dataset is given, with finite $n$ and $T$, the static approach might perform well even if the data were generated by a model not fulfilling the finite-dimension assumption.

Suppose we want to estimate the impulse-response functions of the variables $x_{i t}$ with respect to the shocks $u_{f t}$. Under the dynamic approach, we have to determine $q$ and the maximum lag $S$ for the matrices $\mathbf{A}^{k}(L)$, e.g. by means of information criteria; then estimate the corresponding

$$
\left[S(q+1)^{2}+(q+1) q\right] \frac{n}{q+1}=[S(q+1)+q] n
$$

parameters of the matrices $\mathbf{A}^{k}(L)$ and $\mathbf{R}^{k}$. Under the static approach (1.3), we must determine, $r, q$ and the maximum lag for the matrix $D(L)$, then estimate the corresponding

$$
n r+p r^{2}+r q
$$

parameters $\lambda_{i f}$ and the matrices $\mathbf{D}(L)$ and $\mathbf{R}$. It is easily seen that an a priori assess- 
ment of the relative merits of the two methods is impossible, the situation being much more complicated than the problem we face when deciding which ARMA specification should be chosen for a medium-size stochastic vector.

A simple illustration of the difficulty can be obtained by considering example (1.5) again. In this case the dynamic approach seems definitely superior. Even though a good approximation can be obtained using the static approach, we may argue that there is no good reason to use a moving average when the data have been generated by an autoregression. On the other hand, as the true model is unknown, even if we correctly specify $S$ as 1 in our dynamic model, we end up estimating $n / 2$ unrestricted VAR's of degree 1 for the 2-dimensional vectors $\left(\chi_{i t} \chi_{i+1, t}\right)$, therefore twice the "right" number of parameters.

With these considerations in mind, the static and dynamic methods have been applied to simulated data in several Monte Carlo experiments by Forni et al. (2014). A very short summary of our results is that (i) when the data are generated by infinitedimensional models like (1.5), the estimation of impulse-response functions and predictions by the dynamic method is by far better than those obtained via the static method; (ii) when the data are generated by (1.3), still the dynamic method performs slightly better. Quite similar results are obtained if the data-generating processes are data-driven, i.e. if their coefficients result from applying the static or dynamic approach to a large macroeconomic dataset.

Though not conclusive, the Monte Carlo results in Forni et al. (2014) strongly suggest that the model proposed in the present paper is a competitive specification for dynamic factor models.

\section{Conclusion}

We have argued that assuming a finite-dimensional factor space strongly restricts the applicability of dynamic factor models, as even models as simple as $x_{i t}=\left[a_{i} /\left(1-\alpha_{i} L\right)\right] u_{t}+$ $\xi_{i t}$ are ruled out. On the other hand, without that assumption, only two-sided estimators have been proposed in the literature so far. 
The present paper provides a solution to this problem by means of a feasible autoregressive representation of the high-dimensional common-component vector $\chi_{n t}$. The key result is that if a stochastic vector $\chi_{n t}$ has dimension $n$ and $\operatorname{rank} q$, where $q$ is fixed whereas $n$ is huge and growing, then, under some mild assumptions, for generic values of the parameters, an autoregressive representation for $\chi_{n t}$ can be determined piecewise. We do not need a huge, unfeasible, $n \times n$ VAR, in which each $y_{i t}$ is projected on all $y_{j t-k}, j=1,2, \ldots, n$. A sequence of small $(q+1) \times(q+1)$ VAR's is sufficient.

Using the autoregressive representation of $\chi_{n t}$, we transform the original variables $x_{i t}$ into variables $z_{i t}$ that are governed by a static factor model. All the steps of our construction have a natural counterpart in an estimation procedure.

\section{References}

[1] Alessi, L., Barigozzi, M. and Capasso, M. (2010) Improved penalization for determining the number of factors in approximate factor models. Statistics 6 Probability Letters 80, 1806-1813.

[2] Altissimo, F., R. Cristadoro, M. Forni, M. Lippi and G. Veronese, (2010). New Eurocoin: tracking economic growth in real time, The Review of Economics and Statistics, 92, 1024-1034.

[3] Amengual, D. and M. Watson, (2007) Consistent estimation of the number of dynamic factors in a large $N$ and $T$ panel, Journal of Business and Economic Statistics, 25, 91-96.

[4] Anderson, B. and M. Deistler (2008a). Properties of zero-free transfer function matrices, SICE Journal of Control, Measurement and System Integration 1, 1-9.

[5] Anderson, B. and M. Deistler (2008b). Generalized linear dynamic factor modelsA structure theory, 2008 IEEE Conference on Decision and Control.

[6] Bai, J. (2003). Inferential theory for factor models of large dimensions, Econometrica $71,135-171$. 
[7] Bai, J. and S. Ng (2002). Determining the number of factors in approximate factor models, Econometrica 70, 191-221.

[8] Bai, J. and S. Ng, (2007). Determining the number of primitive shocks in factor models, Journal of Business and Economic Statistics, 25, 52-60.

[9] Bernanke, B. S., and J. Boivin (2003). Monetary policy in a data-rich environment, Journal of Monetary Economics 50, 525546.

[10] Bernanke, B. S., J. Boivin and P. Eliasz (2005). Measuring monetary policy: a factor augmented autoregressive (FAVAR) approach, The Quarterly Journal of Economics 120, 387-422.

[11] Boivin, J. and S. Ng (2006). Are more data always better for factor analysis?, Journal of Econometrics 132, 169-194.

[12] Boivin, J., Giannoni, M.P. and I. Mihov (2009). Sticky Prices and Monetary Policy: Evidence from Disaggregated US Data, American Economic Review 99, 350384.

[13] Brillinger, D. R. (1981). Time Series: Data Analysis and Theory, San Francisco: Holden-Day.

[14] Chamberlain, G. (1983). Funds, factors, and diversification in arbitrage pricing models, Econometrica 51, 1281-1304.

[15] Chamberlain, G. and M. Rothschild (1983). Arbitrage, factor structure and meanvariance analysis in large asset markets, Econometrica 51, 1305-1324.

[16] Corielli, F. and M. Marcellino (2006). Factor based index tracking, Journal of Banking $\&$ Finance 30, 2215-2233.

[17] Cristadoro, R., Forni, M., Reichlin, L. and G. Veronese (2005). A Core Inflation Indicator for the Euro Area, Journal of Money, Credit and Banking, 539-560.

[18] Dunford, N. and J. T. Schwartz, (1988). Linear Operators, Part I. New York: Wiley. 
[19] Eickmeier, S. (2007). Business cycle transmission from the US to Germany-A structural factor approach, European Economic Review 51 521-551.

[20] Favero, C., M. Marcellino, and F. Neglia (2005). Principal components at work: empirical analysis of monetary policy with large data sets, Journal of Applied Econometrics 20, 603-620.

[21] Forni, M. and L. Gambetti (2010b) The dynamic effects of monetary policy: A structural factor model approach, Journal of Monetary Economics 57, 203-216.

[22] Forni, M., M. Hallin, M. Lippi and L. Reichlin (2000). The generalized dynamic factor model: identification and estimation, The Review of Economics and Statistics 82, 540- 554 .

[23] Forni, M., M. Hallin, M. Lippi and L. Reichlin (2004). The generalized dynamic factor model : consistency and rates, Journal of Econometrics 119, 231-255.

[24] Forni, M., M. Hallin, M. Lippi and L. Reichlin (2005). The generalized factor model: one-sided estimation and forecasting, Journal of the American Statistical Association 100, 830-40.

[25] Forni, M., D. Giannone, M. Lippi and L. Reichlin (2009). Opening the black box: structural factor models with large cross-sections, Econometric Theory 25, 1319-1347.

[26] Forni, M., M. Hallin, M. Lippi and P. Zaffaroni (2014). Dynamic factor models with infinite dimensional factor space: estimation and asymptotics, EIEF Working Paper, forthcoming.

[27] Forni, M. and M. Lippi (2001). The generalized dynamic factor model: representation theory, Econometric Theory 17, 1113-1341.

[28] Forni, M. and M. Lippi (2011). The generalized dynamic factor model: one-sided representation results, Journal of Econometrics 163, 23-28. 
[29] Geweke, J. (1977). The dynamic factor analysis of economic time series, in D.J. Aigner and A.S. Goldberger, Eds., Latent Variables in Socio-Economic Models. Amsterdam: North Holland.

[30] Giannone, D., L. Reichlin and L. Sala (2005). Monetary policy in real time, in NBER Macroeconomic Annual 2004, eds. M. Gertler and K. Rogoff, Cambridge, MA: MIT Press, 161200.

[31] Giannone, D., Reichlin, L. and D. Small (2008). Nowcasting: The real-time informational content of macroeconomic data, Journal of Monetary Economics 55, 665-676.

[32] Hallin M. and R. Liška (2007). Determining the number of factors in the general dynamic factor model, Journal of the American Statistical Association 102, 603617.

[33] Hallin M., Ch. Mathias, H. Pirotte and D. Veredas (2011). Market liquidity as dynamic factors, Journal of Econometrics 163, 42-50.

[34] Hannan, E.J. (1970). Multiple Time Series, New York: Wiley.

[35] Ludvigson, S.C. and S. Ng (2007). The empirical risk-return relation: a factor analysis approach, Journal of Financial Economics 83, 171-222.

[36] Ludvigson, S.C. and S. Ng (2009). Macro factors in bond risk premia, The Review of Financial Studies 22, 5027-5067.

[37] Miamee, A.G. and M. Pourahmadi (1987). Degenerate multivariate stationary processes. Basicity, past and future, and autoregressive representation, Sankhya Ser. A 49, 316-334.

[38] Onatski, A. (2009). Testing hypotheses about the number of factors in large factor models, Econometrica 77, 1447-1479.

[39] Pourahmadi, M. (2001). Foundations of Time Series Analysis and Prediction Theory, New York: Wiley. 
[40] Quah, D. and T.J. Sargent (1993). A Dynamic Index Model for Large Cross Sections, in Business Cycles, Indicators and Forecasting, National Bureau of Economic Research.

[41] Rozanov, Yu. (1967). Stationary Random processes, San Francisco: Holden Day.

[42] Salehi, H. (1981). The Continuation of Wiener's work on $q$-variate linear prediction and its extension to infinite-dimensional spaces. In P. Masani, Ed., Norbert Wiener: Collected Work, Volume III, MIT Press, 307-338.

[43] Sargent, T.J. and C.A. Sims (1977). Business cycle modelling without pretending to have too much a priori economic theory, in C.A. Sims, Ed., New Methods in Business Research, Minneapolis: Federal Reserve Bank of Minneapolis.

[44] Stock, J.H. and M.W. Watson (2002a). Macroeconomic Forecasting Using Diffusion Indexes, Journal of Business and Economic Statistics 20, 147-162.

[45] Stock, J.H. and M.W. Watson (2002b). Forecasting using principal components from a large number of predictors, Journal of the American Statistical Association 97, 1167-1179.

[46] Stock, J.H., and M.W. Watson (2005). Implications of dynamic factor models for VAR analysis, NBER Working Papers no. 11467.

[47] van der Waerden, B.L. (1953). Modern Algebra, Vol. I. New York: Frederick Ungar.

\section{Appendix}

\section{A Proof of Proposition 1}

A polynomial of the form

$$
p(L)=a_{0}+a_{1} L+\cdots+a_{r} L^{r},
$$

where the coefficients $a_{k}$ are either scalar or matrices, is said to have degree not greater than $r$; we say that $p(L)$ has degree $r$ if $a_{r} \neq 0$. We need some preliminary results. 
Lemma A.1 Assume that $\mathbf{v}_{t}=\left(v_{1 t} v_{2 t} \ldots v_{q_{t}}\right)$ is orthonormal white noise and let

$$
y_{i t}=\gamma_{i 1}(L) v_{1 t}+\gamma_{i 2}(L) v_{2 t}+\cdots+\gamma_{i q}(L) v_{q t}
$$

for $i=1,2, \ldots, n$, where the filters $\gamma_{i f}(L)$ are square-summable. In compact form,

$$
\mathbf{y}_{t}=\boldsymbol{\Gamma}(L) \mathbf{v}_{t}
$$

where $\boldsymbol{\Gamma}(L)$ is $n \times q$. For $R \geq 1$ consider the $n R$-dimensional stack

$$
\mathbf{Y}_{t}=\left(\begin{array}{llll}
\mathbf{y}_{t}^{\prime} & \mathbf{y}_{t-1}^{\prime} & \cdots & \mathbf{y}_{t-R+1}^{\prime}
\end{array}\right)^{\prime}
$$

and the $1 \times q$ filter

$$
\mathbf{W}(L)=\left(\begin{array}{llll}
\beta_{1}(L) & \beta_{2}(L) & \cdots & \beta_{n}(L)
\end{array}\right) \boldsymbol{\Gamma}(L)
$$

where $\beta_{i}(L)$ is a finite-degree polynomial in $L, i=1,2, \ldots, n$. The entries of $\mathbf{Y}_{t}$ are linearly dependent if and only if there exist polynomials $\beta_{i}(L)$ of degree not greater than $R-1$, with $\beta_{i}(L) \neq 0$ for some $i$, such that $\mathbf{W}(L)=\mathbf{0}$. Equivalently, the entries of $\mathbf{Y}_{t}$ are linearly independent if and only if $\mathbf{W}(L)=\mathbf{0}$ implies that either $\beta_{i}(L)=0$ for all $i$ or that the degree of $\beta_{i}(L)$ is greater than $R-1$ for some $i$.

Proof. If the entries of $\mathbf{Y}_{t}$ are linearly dependent, there exists

$$
\boldsymbol{\alpha}=\left(\alpha_{01} \cdots \alpha_{0, n} ; \alpha_{11} \cdots \alpha_{1, n} ; \cdots ; \alpha_{R-1,1} \cdots \alpha_{R-1, n}\right) \neq \mathbf{0}
$$

such that

$$
\boldsymbol{\alpha}\left(\mathbf{y}_{t}^{\prime} \mathbf{y}_{t-1}^{\prime} \cdots \mathbf{y}_{t-R+1}^{\prime}\right)^{\prime}=\mathbf{0}
$$

that is, setting $\boldsymbol{\alpha}_{k}=\left(\begin{array}{lll}\alpha_{k 1} & \cdots & \alpha_{k, n}\end{array}\right)$,

$$
\begin{aligned}
\boldsymbol{\alpha}_{0} \boldsymbol{\Gamma}(L) \mathbf{v}_{t}+\boldsymbol{\alpha}_{1} \boldsymbol{\Gamma}(L) v_{t-1}+\cdots+ & \boldsymbol{\alpha}_{R-1} \boldsymbol{\Gamma}(L) \mathbf{v}_{t-R+1}= \\
& \left(\boldsymbol{\alpha}_{0}+\boldsymbol{\alpha}_{1} L+\cdots+\boldsymbol{\alpha}_{R-1} L^{R-1}\right) \boldsymbol{\Gamma}(L) \mathbf{v}_{t}=0 .
\end{aligned}
$$

Because $\mathbf{v}_{t}$ is orthonormal white noise, (A.2) implies that

$$
\left(\boldsymbol{\alpha}_{0}+\boldsymbol{\alpha}_{1} L+\cdots+\boldsymbol{\alpha}_{R-1} L^{R-1}\right) \boldsymbol{\Gamma}(L)=\mathbf{0}
$$


that is, setting $\beta_{i}(L)=\alpha_{0 i}+\alpha_{1 i} L+\cdots+\alpha_{R-1, i} L^{R-1}, i=1,2, \ldots, n$,

$$
\left(\beta_{1}(L) \beta_{2}(L) \cdots \beta_{q+1}(L)\right) \boldsymbol{\Gamma}(L)=\mathbf{0}
$$

Since $\boldsymbol{\alpha} \neq \mathbf{0}, \beta_{i}(L) \neq 0$ for some $i$. Conversely, starting with (A.3), where the degree of $\beta_{i}(L)$ is not greater than $R-1$ and $\beta_{i}(L) \neq 0$ for some $i$, we easily obtain an $\boldsymbol{\alpha} \neq \mathbf{0}$ such that (A.1) holds.

Q.E.D.

Lemma A.2 Assume that $\mathbf{v}_{t}=\left(v_{1 t} v_{2 t} \ldots v_{q t}\right)$ is orthonormal white noise and

$$
y_{i t}=p_{i 1}(L) v_{1 t}+p_{i 2}(L) v_{2 t}+\cdots+p_{i q}(L) v_{q t}
$$

with

$$
p_{i f}(L)=p_{i f, 0}+p_{i f, 1} L+\cdots+p_{i f, r} L^{r}
$$

for $i=1,2, \ldots, q+1, f=1,2, \ldots, q$. In compact form,

$$
\mathbf{y}_{t}=\mathbf{P}_{0} \mathbf{v}_{t}+\mathbf{P}_{1} \mathbf{v}_{t-1}+\cdots+\mathbf{P}_{r} \mathbf{v}_{t-r}=\mathbf{P}(L) \mathbf{v}_{t}
$$

where the matrices $\mathbf{P}_{k}$ are $(q+1) \times q$. Let $R=r q$. Assume that the entries of the stack $\mathbf{Y}_{t}=\left(\begin{array}{llll}\mathbf{y}_{t-1}^{\prime} & \mathbf{y}_{t-2}^{\prime} & \cdots & \mathbf{y}_{t-R}^{\prime}\end{array}\right)^{\prime}$ are linearly independent. Then,

$$
\mathbf{y}_{t}=\mathbf{H}_{1} \mathbf{y}_{t-1}+\cdots+\mathbf{H}_{R} \mathbf{y}_{t-R}+\mathbf{P}_{0} \mathbf{v}_{t}
$$

for some $(q+1) \times(q+1)$ matrices $\mathbf{H}_{k}$.

Proof. Consider the stack

$$
\mathbf{Y}_{t-1}=\left(\begin{array}{llll}
\mathbf{y}_{t-1}^{\prime} & \mathbf{y}_{t-2}^{\prime} & \cdots & \mathbf{y}_{t-R}^{\prime}
\end{array}\right)^{\prime}=\mathcal{P}_{R}\left(\mathbf{v}_{t-1}^{\prime} \mathbf{v}_{t-2}^{\prime} \cdots \mathbf{v}_{t-R-r}^{\prime}\right)^{\prime}
$$

where

$$
\mathcal{P}_{R}=\left(\begin{array}{ccccccc}
\mathbf{P}_{0} & \mathbf{P}_{1} & \cdots & \mathbf{P}_{r} & \mathbf{0} & \cdots & \mathbf{0} \\
\mathbf{0} & \mathbf{P}_{0} & \cdots & \mathbf{P}_{r-1} & \mathbf{P}_{r} & \cdots & \mathbf{0} \\
\vdots & & & & & \ddots & \\
\mathbf{0} & \mathbf{0} & \cdots & & & \cdots & \mathbf{P}_{r}
\end{array}\right) .
$$

The matrix $\mathcal{P}_{R}$ is $(q+1) R \times q(R+r)$. Setting $R=r q, \mathcal{P}_{R}$ is square. By assumption, the entries $\mathbf{Y}_{t-1}$ are linearly independent. Thus the matrix $\mathcal{P}_{R}$ is non singular, so that $\left(\mathbf{v}_{t-1}^{\prime} \mathbf{v}_{t-2}^{\prime} \cdots \mathbf{v}_{t-R-r}^{\prime}\right)^{\prime}=\mathcal{P}_{R}^{-1}\left(\mathbf{y}_{t-1}^{\prime} \mathbf{y}_{t-2}^{\prime} \cdots \mathbf{y}_{t-R}^{\prime}\right)^{\prime}$. Substituting $\mathbf{v}_{t-1}, \mathbf{v}_{t-2} \cdots, \mathbf{v}_{t-r}$ into (A.5), we get (A.6).

Q.E.D. 
Lemma A.3 Rewrite (4.1) in compact form

$$
\mathbf{y}_{t}=\mathbf{E}(L) \mathbf{v}_{t}
$$

where

$$
e_{i f}(L)=\frac{c_{i f}(L)}{d_{i f}(L)}=\frac{c_{i f, 0}+c_{i f, 1} L+\cdots+c_{i f, s_{1}} L^{s_{1}}}{1+d_{i f, 1} L+\cdots+d_{i f, s_{2}} L^{s_{2}}}
$$

for $i=1,2, \ldots, q+1, f=1,2, \ldots, q$. Let $S=s_{1} q+s_{2} q^{2}$. For generic values of the parameters, the entries of the stack $\left(\mathbf{y}_{t}^{\prime} \mathbf{y}_{t-1}^{\prime} \cdots \mathbf{y}_{t-S+1}^{\prime}\right)^{\prime}$ are linearly independent.

Proof. Using the notation of Section 4 , let $\mu=(q+1) q\left(s_{1}+s_{2}+1\right)$. Denote by $p=\left(p_{1} p_{2} \cdots p_{\mu}\right)$ the $\mu$-dimensional vectors of $\Pi^{q+1}$ (the entries of $p$ are the parameters $c$ and $d$ ). In this proof, we deal with scalar polynomials in $L$

$$
a_{0}+a_{1} L+\cdots+a_{r} L^{r}
$$

where the coefficients $a_{m}$ are polynomials in the parameters, of the form

$$
\sum_{k_{1}+k_{2}+\cdots+k_{\mu} \leq K} \alpha_{k_{1}, k_{2}, \ldots, k_{\mu}} p_{1}^{k_{1}} p_{2}^{k_{2}} \cdots p_{\mu}^{k_{\mu}}
$$

Because $\Pi^{q+1}$ is the closure of an open set in $\mathbb{R}^{\mu}$, the polynomial (A.8) is generically non zero in $\Pi^{q+1}$ if and only if at least one coefficient $\alpha_{k_{1}, k_{2}, \ldots, k_{\mu}}$ is non zero. Note also that (A.8) can be rewritten as a polynomial in one of the variables, $p_{1}$ for example,

$$
A_{0} p_{1}^{M}+A_{1} p_{1}^{M-1}+\cdots+A_{M}
$$

where the coefficients $A_{j}$ are polynomials in $p_{2}, \ldots, p_{\mu}$, and that (A.8) is generically non zero in $\Pi^{q+1}$ if and only if at least one of the coefficients $A_{j}$ in (A.9) is generically non zero.

By Lemma A.1, we must prove that, for generic values in $\Pi^{q+1}$, if

$$
\left(\beta_{1}(L) \quad \beta_{2}(L) \quad \cdots \quad \beta_{n}(L)\right) \mathbf{E}(L)=\mathbf{0}
$$

where $\beta_{i}(L)$ is a finite-degree polynomial and $\beta_{i}(L) \neq 0$ for some $i$, then the degree of $\beta_{i}(L)$ is greater than $S-1$ for some $i$. Let $\mathbf{E}_{q}(L)$ be the square submatrix obtained by dropping $\mathbf{E}(L)$ 's last row. We can write

$$
\operatorname{det}\left(\mathbf{E}_{q}(L)\right)=h(L) / \prod_{i, f=1}^{q} d_{i f}(L),
$$


where numerator and denominator have degree not greater than $S_{1}=q s_{1}+\left(q^{2}-\right.$ q) $s_{2}$ and $S_{2}=s_{2} q^{2}$, respectively. The coefficient of $L^{S_{2}}$ in the denominator is the product $\prod_{i, f=1}^{q} d_{i f, s_{2}}$ and is therefore generically non zero. The coefficient of $L^{S_{1}}$ in the numerator contains the term

$$
c_{11, s_{1}} c_{22, s_{1}} \cdots c_{q q, s_{1}} \prod_{\substack{i, f=1, q \\ i \neq f}} d_{i f, s_{2}}
$$

and no other term with the same exponents for the $c$ 's and the d's. Thus, generically, numerator and denominator in (A.11) have degrees $S_{1}$ and $S_{2}$, respectively.

Using the same argument, the $(i, f)$ entry of the adjoint matrix of $\mathbf{E}_{q}(L)$ can be written as

$$
h_{i f}(L) / \prod_{\substack{h, k=1, \ldots q \\ h \neq f, k \neq i}} d_{h k}(L)
$$

where generically the degrees of the numerator and the denominator are $S_{3}=(q-$ 1) $s_{1}+\left[(q-1)^{2}-(q-1)\right] s_{2}$ and $S_{4}=(q-1)^{2} s_{2}$, respectively. Thus, the matrix $\mathbf{E}_{q}(L)$ is generically invertible, as a matrix of rational functions in $L$, and the entries of $\left[\mathbf{E}_{q}(L)\right]^{-1}$ can be written as

$$
h_{i f}(L) \prod_{\substack{h, j=1, \ldots, q \\ h=f \text { or } k=i}} d_{h k}(L) / h(L)=\tilde{h}_{i f}(L) / h(L),
$$

where generically the degrees of the numerator and the denominator are $S_{5}=(q-$ 1) $s_{1}+\left(q^{2}-(q-1)\right) s_{2}$ and $S_{6}=q s_{1}+\left(q^{2}-q\right) s_{2}$, respectively.

Consider now the system of equations

$$
\left(\rho_{1}(L) \rho_{2}(L) \cdots \rho_{q}(L)\right) \mathbf{E}_{q}(L)=-\left(e_{q+1,1}(L) e_{q+1,2}(L) \cdots e_{q+1, q}(L)\right)
$$

in the unknown rational functions $\rho_{k}(L)$. Generically, the system has the unique solution

$$
\left(\tau_{1}(L) \tau_{2}(L) \cdots \tau_{q}(L)\right)=-\left(e_{q+1,1}(L) e_{q+1,2}(L) \cdots e_{q+1, q}(L)\right)\left[\mathbf{E}_{q}(L)\right]^{-1} .
$$

We have

$\tau_{k}(L)=-\sum_{i=1}^{q} \frac{c_{q+1, i}(L) \tilde{h}_{i k}(L)}{d_{q+1, i}(L) h(L)}=-\frac{\sum_{i=1}^{q}\left[c_{q+1, i}(L) \tilde{h}_{i k}(L) \prod_{\substack{j=1, \ldots, q \\ j \neq i}} d_{q+1, j}(L)\right]}{h(L) \prod_{i=1}^{q} d_{q+1, i}(L)}=-\frac{\nu_{k}(L)}{\delta(L)}$, 
where generically both $\nu_{k}(L)$ and $\delta(L)$ are polynomials of degree $S=q s_{1}+q^{2} s_{2}$. Moreover, for generic values of the parameters, $\nu_{k}(L)$ and $\delta(L)$ have no roots in common. To show this, recall that the polynomials

$$
\nu_{k}(L)=\nu_{k, S} L^{S}+\nu_{k, S-1} L^{S-1}+\ldots+\nu_{k, 0} \quad \text { and } \quad \delta(L)=\delta_{S} L^{S}+\delta_{S-1} L^{S-1}+\ldots+\delta_{0},
$$

both of degree $S$, have roots in common if and only if their resultant vanishes. That resultant is a polynomial in the coefficients $\nu_{k, j}$ and $\delta_{j}$, involving the term $\nu_{k, S}^{S} \delta_{0}^{S}$ (see van der Waerden 1953, pp. 83-5). All other terms contain powers $\nu_{k, S}^{S-h}$ with $0<h \leq S$. We have

$\nu_{k, S}^{S} \delta_{0}^{S}=\left[\sum_{i=1}^{q} c_{q+1, i, s_{1}} \tilde{h}_{i k, g} \prod_{\substack{j=1, \ldots, q \\ j \neq i}} d_{q+1, j, s_{2}}\right]^{S} h(0)^{S}=c_{q+1,1, s_{1}}^{S}\left[\tilde{h}_{1 k, S_{5}}^{S} \prod_{j=2, \ldots, q} d_{q+1, j, s_{2}}^{S} h(0)^{S}\right]+\ldots$,

where $\tilde{h}_{i k, S_{5}}$ is the coefficient of order $S_{5}$ of $\tilde{h}(L)$. Note that $h(L)$ and $\tilde{h}_{i f}(L)$ do not contain any of the parameters $c_{q+1, i, h}$. As a consequence, all other terms in (A.12) and in the resultant of $\nu_{k}(L)$ and $\delta(L)$ contain powers $c_{q+1, i, s_{1}}^{S-h}$, with $0<h \leq S$. Thus the three-term product within square brackets in the right-hand side of (A.12) is the coefficient of $c_{q+1,1, s_{1}}^{S}$ in the representation of the resultant as a polynomial in $c_{q+1,1, s_{1}}$. As each of the three terms is generically non zero, the coefficient is generically non zero, so that the resultant is generically non zero.

Suppose now that the polynomials $\beta_{k}(L)$ 's are such that (A.10) holds, that is

$$
\left(\beta_{1}(L) \beta_{2}(L) \cdots \beta_{q}(L)\right) \mathbf{E}_{q}(L)=-\beta_{q+1}(L)\left(e_{q+1,1}(L) e_{q+1,2}(L) \cdots e_{q+1, q}(L)\right) .
$$

Because the matrix $\mathbf{E}_{q}(L)$ is generically non singular, as a matrix of rational functions, $\beta_{q+1}(L)=0$ implies $\beta_{i}(L)=0$ for all $i=1,2, \ldots, q+1$. Assuming that $\beta_{q+1}(L) \neq 0$, we have

$$
\tau_{k}(L)=-\frac{\beta_{k}(L)}{\beta_{q+1}(L)} .
$$

The results above on $\tau_{k}(L)$ imply that generically the degree of $\beta_{q+1}(L)$ and $\beta_{k}(L)$ is at least $S$.

Q.E.D. 
We now can proceed with the proof of Proposition 1. Rewrite (A.7) as

$$
\left(\begin{array}{cccc}
h_{1}(L) & 0 & \cdots & 0 \\
0 & h_{2}(L) & \cdots & 0 \\
& & \ddots & \\
0 & 0 & \cdots & h_{q+1}(L)
\end{array}\right) \mathbf{y}_{t}=\mathbf{G}(L) \mathbf{v}_{t}
$$

where

$$
h_{i}(L)=\prod_{f=1}^{q} d_{i f}(L), \quad g_{i f}(L)=c_{i f}(L) \prod_{\substack{f=1, \ldots, q \\ f \neq i}} d_{i f}(L) .
$$

Let us focus on the moving average on the right-hand side. The polynomial matrix $\mathbf{G}(L)$ has degree not greater than $\tilde{S}=s_{1}+s_{2}(q-1)$. Suppose that

$$
\left(\beta_{1}(L) \quad \beta_{2}(L) \quad \cdots \quad \beta_{q+1}(L)\right) \mathbf{G}(L)=\mathbf{0}
$$

where the degree of $\beta_{j}(L)$ is not greater than $\tilde{S} q-1$. This implies that

$$
\left(\beta_{1}(L) h_{1}(L) \quad \beta_{2}(L) h_{2}(L) \quad \cdots \quad \beta_{q+1}(L) h_{q+1}(L)\right) \mathbf{E}(L)=\mathbf{0} .
$$

The polynomials $\beta_{j}(L) h_{j}(L)$ have degrees not greater than $\tilde{S} q-1+s_{2} q=s_{1} q+s_{2} q^{2}-1$. Lemmas A.3 and A.1 imply that generically $\beta_{i}(L) h_{i}(L)=0$ for all $i=1,2, \ldots, q+1$. Because $h_{i}(L) \neq 0$ for all $i$, then generically (A.15) implies $\beta_{i}(L)=0$ for all $i$. Using Lemma A.2, $\mathbf{G}(L) \mathbf{v}_{t}$ generically has an autoregressive representation of degree $s_{1} q+$ $s_{2} q(q-1)$, so that, by (A.13)-(A.14), $\mathbf{y}_{t}$ generically has an autoregressive representation

$$
\mathbf{y}_{t}=\mathbf{K}_{1} \mathbf{y}_{t-1}+\mathbf{K}_{2} \mathbf{y}_{t-2}+\cdots+\mathbf{K}_{S} \mathbf{y}_{t-S}+\mathbf{E}(0) \mathbf{v}_{t}
$$

of degree $S=s_{1} q+s_{2} q^{2}$. Moreover, Lemma A.3 proves that generically the components of the stack

$$
\left(\begin{array}{llll}
\mathbf{y}_{t-1}^{\prime} & \mathbf{y}_{t-2}^{\prime} & \cdots & \mathbf{y}_{t-S}^{\prime}
\end{array}\right)^{\prime}
$$

are independent. The uniqueness part of the proposition follows.

Q.E.D. 